\title{
Partially Flagged Parallel Manipulators: Singularity Charting and Avoidance
}

\author{
Maria Alberich-Carramiñana, Marçal Garolera, Federico Thomas, Member, IEEE, and Carme Torras, Member, IEEE
}

\begin{abstract}
There are only three 6-SPS parallel manipulators with triangular base and platform, i.e., the octahedral, the flagged, and the partially flagged, which are studied in this paper. The forward kinematics of the octahedral manipulator is algebraically intricate, while those of the other two can be solved by three trilaterations. As an additional nice feature, the flagged manipulator is the only parallel platform for which a cell decomposition of its singularity locus has been derived. Here, we prove that the partially flagged manipulator also admits a well-behaved decomposition, technically called a stratification, some of whose strata are not topological cells, however. Remarkably, the adjacency diagram of the 5-D and 6-D strata (which shows what 5-D strata are contained in the closure of a 6-D one) is the same as for the flagged manipulator. The availability of such a decomposition permits devising a redundant 7-SPS manipulator, combining two partially flagged ones, which admits a control strategy that completely avoids singularities. Simulation results support these claims.
\end{abstract}

Index Terms-Configuration space, kinematics singularities, parallel manipulators, redundant manipulators, robot design.

\section{INTRODUCTION}

$\mathbf{M}$ ANY efforts have been devoted to the singularity analysis of the Stewart-Gough platform and its specializations [1]-[3]. Most of the works characterize singularities analytically, without providing much insight on their topological arrangement in the configuration space of the platform with respect to the base, which has been singled out as an important open problem in parallel robotics [4]. Indeed, one of today's main shortcomings of parallel platforms is that they are forced to operate in reduced workspaces in order to keep away from singular configurations. A complete knowledge of the arrangement of singularity hypersurfaces in configuration space would be most useful for manipulator design, including the use of redundant actuators [5] or joint coupling [6] to eliminate certain

Manuscript received August 7, 2008; revised February 6, 2009 and March 5, 2009. First version published May 2, 2009; current version published July 31, 2009. This paper was recommended for publication by Associate Editor I. Bonev and Editor F. Park upon evaluation of the reviewers' comments. This work was supported in part by the Catalan Research Commission. The work of M. Alberich-Carramiñana was supported by DGICYT under Project MTM200614234-C02-02

M. Alberich-Carramiñana is with the Institut de Robòtica i Informàtica Industrial, Spanish Scientific Research Council-Universitat Politècnica de Catalunya, 08028 Barcelona, Spain, and also with the Department of Applied Mathematics I, Universitat Politècnica de Catalunya, 08028 Barcelona, Spain (e-mail: maria.alberich@upc.edu).

M. Garolera was with the Institut de Robòtica i Informàtica Industrial, Spanish Scientific Research Council-Universitat Politècnica de Catalunya, 08028 Barcelona, Spain. He is now with Google, Inc., Dublin 4, Ireland (e-mail: m.garolera@gmail.com).

F. Thomas and C. Torras are with the Institut de Robòtica i Informàtica Industrial, Spanish Scientific Research Council-Universitat Politècnica de Catalunya, 08028 Barcelona, Spain (e-mail: thomas@iri.upc.edu; torras@iri.upc.edu).

Digital Object Identifier 10.1109/TRO.2009.2018970 singularities, as well as to plan trajectories away from singularities or crossing them in a controlled way, thus allowing the robot to operate in much larger workspaces.

By specializations of the Stewart-Gough platform we mean 6-SPS designs in which some $S$ joints merge in either the platform, the base, or both. An interesting subset of such specializations is the one in which there are only three multiple spherical pairs in both the platform and the base, which thus become triangular. These are often referred to as 3-3 manipulators. There are three architectures of this type (see Fig. 1), namely the well-known octahedral manipulator [7], [8], the basic flagged manipulator [9]-[13], and the, so far, barely known partially flagged manipulator, which is the object of this paper.

The name "flagged" comes from the fact that one such manipulator is characterized by the relation between two flags adequately placed on its platform and base: a flag being a triple (point, line, and plane) with the point contained in the line, which, in turn, lies on the plane. The interesting property of such a manipulator is that its singularity locus admits a cell decomposition inherited from that of the flag manifold [13], which can be described in terms of incidences between the elements (point, line, and plane) of the two flags.

Analogously, as we will show in this paper, a partially flagged manipulator can be characterized by the relation between one flag and one partial flag, i.e., one in which the point is not contained in the line. Moreover, it will be proven that its singularity locus admits also a well-behaved decomposition, whose components are no longer topological cells, however.

As mentioned earlier, research on parallel manipulators has led to the elimination of singularities by adding actuators either in an existing or in an added leg, i.e., by introducing redundancy. Merlet has outlined the key concepts to be considered while designing and using a redundant parallel manipulator [14]. Since adding a redundant leg may decrease the dimension of the singularity space [15], some research has been carried out to determine where to locate this leg to effectively decrease or even eliminate a singularity surface [16]. The result has been several successful implementations of redundant parallel manipulators with extra legs [17], [18]. It is worth noting here that the idea of using redundant actuators is closely related to that of adding extra sensors to obtain unique closed-form solutions for the forward kinematics of parallel manipulators (see, e.g., [19] and references therein). Thus, the literature on the location of extra sensors in parallel manipulators is also an important source of inspiration to decide where to locate extra actuators.

In a 7-leg parallel manipulator, by switching which leg remains passive, the distribution of singularities across the configuration space of the platform with respect to the base 


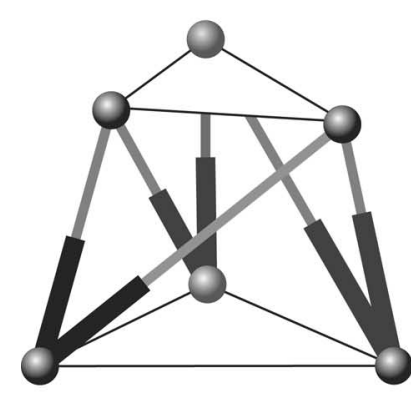

(a)

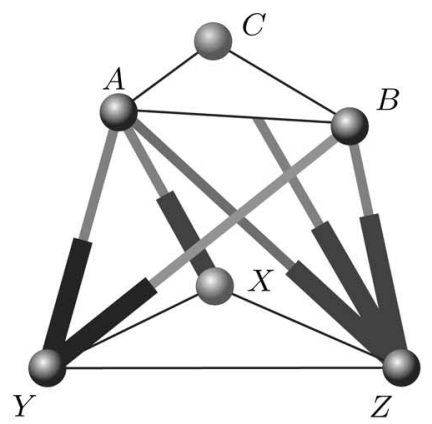

(b)

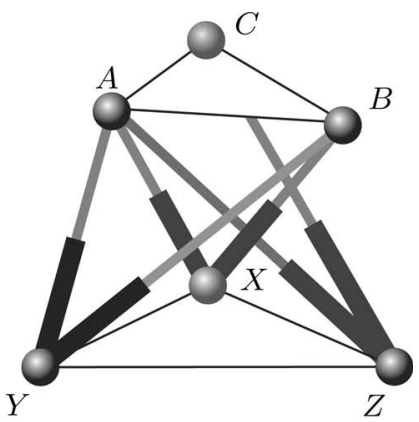

(c)

Fig. 1. The three architectures for the 3-3 parallel manipulators. (a) Octahedral. (b) Flagged. (c) Partially flagged.

(C-space, for short) changes, and one can exploit this change for singularity avoidance. To this aim, it is necessary to have a complete and precise characterization of the singularity loci of the involved manipulators which, in general, is not available.

In our case, having the decomposition of the singularity loci of partially flagged manipulators has allowed us to devise a redundant 7-SPS architecture, combining two partially flagged manipulators, which admits a control strategy that completely avoids singularities. The underlying idea is as follows. At the higher dimensional level, the C-spaces of both manipulators share a very similar structure with an identical adjacency graph between the eight 6-D component regions, corresponding to the eight forward kinematic solutions or assembly modes, and the twelve 5-D regions, corresponding to singular hypersurface patches. By adjacency between two subsets we mean that one subset is contained in the closure of the other. However, these earlier mentioned regions are not equally located for both manipulators. Then, switching actuation from one SPS chain to another implies converting one manipulator into another, thus changing the location of the 5-D regions. The 7-SPS architecture proposed has been designed so that 5-D regions (singularity loci) of the two manipulators have at most a 4-D intersection. This means that the $\mathrm{C}$-space of the resulting manipulator with switched control has singularity loci of at most dimension 4 , which can easily be avoided in practice. Note that, to avoid singularities, manipulators are often made to operate within just one 6-D component region. By adding an extra actuator and using switched control, the operation workspace thus becomes considerably enlarged, thereby permitting free motion between the eight 6-D component regions without having to cross any singular hypersurface.

This paper is structured as follows. The next section introduces the partially flagged 3-3 architecture and describes its forward kinematics as a trilaterable manipulator. Section III studies the topology of its singularity locus as inherited from a new stratification of the flag manifold (the variety parameterizing all the possible placements of a flag in the real 3-D projective space), which is considerably different from the classical one. The adjacency diagram of the strata of dimension 6 (nonsingular) and dimension 5 (singular) is worked out in detail. Section IV discusses the design of the 7-SPS redundant manipulator and the switching strategy that permits the avoidance of singularities. Some simulation results on such redundant manipulator are presented in Section V. Finally, Section VI provides some conclusions and points that deserve further attention.

\section{Forward Kinematics of THE PARTIALly FlagGed 3-3 MANIPULATOR}

Let us consider the set of 6-legged manipulators whose leg end-points merge into three multiple spherical joints both in the base and the platform. There are only three possible architectures for this kind of manipulators, also known as 3-3 manipulators (see Fig. 1). One of them corresponds to the well-known octahedral manipulator [see Fig. 1(a)] whose forward kinematics is quite involved as it amounts to solving an eighth-degree polynomial, leading to 16 solutions or assembly modes [8], [20], [21]. On the contrary, the forward kinematics of the other two can be solved by a sequence of three consecutive trilaterations, leading to eight solutions [10]-[12]. The flagged 3-3 manipulator [see Fig. 1(b)] was thoroughly studied in [13]. In what follows, we concentrate our attention on the forward kinematics of the partially flagged 3-3 manipulator in Fig. 1(c). Using the notation in this figure, the lengths of the six actuators $d_{1}, \ldots, d_{6}$ satisfy the following relations:

$$
\begin{aligned}
& d_{1}^{2}=\|A X\|^{2}, \quad d_{2}^{2}=\|A Y\|^{2}, \quad d_{3}^{2}=\|A Z\|^{2} \\
& d_{4}^{2}=\|B X\|^{2}, \quad d_{5}^{2}=\|B Y\|^{2}, \quad d_{6}^{2}=\|C Z\|^{2} .
\end{aligned}
$$

The configuration of the manipulator will be specified as an element of $\mathbb{R}^{3} \times \mathrm{SO}(3)$, i.e., a translation $(x, y, z)$ of the platform and a rotation $\left(\theta_{x}, \theta_{y}, \theta_{z}\right)$ centered in $A$. The relation between the linear actuators' velocities and the platform's velocity vector is

$$
\left(\begin{array}{cccccc}
d_{1} & 0 & 0 & 0 & 0 & 0 \\
0 & d_{2} & 0 & 0 & 0 & 0 \\
0 & 0 & d_{3} & 0 & 0 & 0 \\
0 & 0 & 0 & d_{4} & 0 & 0 \\
0 & 0 & 0 & 0 & d_{5} & 0 \\
0 & 0 & 0 & 0 & 0 & d_{6}
\end{array}\right)\left(\begin{array}{c}
\dot{d}_{1} \\
\dot{d}_{2} \\
\dot{d}_{3} \\
\dot{d}_{4} \\
\dot{d}_{5} \\
\dot{d}_{6}
\end{array}\right)
$$




$$
=\left(\begin{array}{cc}
A X & 0 \\
A Y & 0 \\
A Z & 0 \\
B X & A B \times A X \\
B Y & A B \times A Y \\
C Z & A C \times A Z
\end{array}\right)\left(\begin{array}{c}
\dot{x} \\
\dot{y} \\
\dot{z} \\
\dot{\theta_{x}} \\
\dot{\theta_{y}} \\
\dot{\theta_{z}}
\end{array}\right) .
$$

The singularities of the manipulator correspond to the singularities in either of the two Jacobians [22]. The first matrix is singular when any of the lengths of the actuators is zero, i.e., in the limit of the control variables. These are type-I or inverse kinematics singularities. We will now focus on type-II or forward kinematics singularities, namely those originated by the second Jacobian, which loses its full rank when either

$$
\left(\begin{array}{c}
A X \\
A Y \\
A Z
\end{array}\right) \text { or }\left(\begin{array}{c}
A B \times A X \\
A B \times A Y \\
A C \times A Z
\end{array}\right)
$$

are singular. The left matrix is singular when the tetrahedron $A X Y Z$ has volume zero, which can be understood as $A$ lying on the plane $X Y Z$. In the second matrix, $A B \times A X$ and $A B \times A Y$ are linearly dependent if and only if the tetrahedron $A B X Y$ degenerates, i.e., the line going through $A$ and $B$ and the line going through $X$ and $Y$ either intersect or are parallel. Finally, the condition that $A C \times A Z$ is dependent of the other two vectors can be written as $A B C Z$ having null volume, meaning that $Z$ is in the plane $A B C$.

To study these singularities, we will use the following features of the manipulator: a point $v=Z$, a line $l=X Y$, and a plane $p=X Y Z$ in the base, and a point $v^{*}=A$, a line $l^{*}=A B$, and a plane $p^{*}=A B C$ in the platform. Then, the degeneration of the tetrahedra $A X Y Z, A B X Y$, and $A B C Z$ corresponds to $v^{*} \subset p, l \cap l^{*} \neq 0$, and $v \subset p^{*}$, respectively.

Note that the features satisfy

$$
\begin{gathered}
v^{*} \subset l^{*} \subset p^{*} \\
v \nsubseteq l \subset p \quad \text { and } \quad v \subset p .
\end{gathered}
$$

This fact will be used in the next section to attach a flag (a point contained in a line contained in a plane) to the platform and study its incidences with $v, l$, and $p$ to derive the topology of the singularities.

It is worth mentioning that, in the case of a flagged 3-3 manipulator, the singularities correspond to the degeneration of the tetrahedra $A X Y Z, A B Y Z$, and $A B C Z$. Thus, in this case, $l=Y Z$, the other features remaining the same, which leads to $v^{*} \subset l^{*} \subset p^{*}$ and $v \subset l \subset p$, and therefore, flags could be attached to both the base and the platform [13].

\section{Topology OF Singularities of THE Partially FLAGGED 3-3 MANIPULATOR}

Consider a partially flagged 3-3 parallel manipulator, whose features are the point $v$, the line $l$, and the plane $p$ on the base, and the point $v^{*}$, the line $l^{*}$, and the plane $p^{*}$ on the platform, satisfying $v^{*} \subset l^{*} \subset p^{*}, v \nsubseteq l \subset p$, and $v \subset p$. In order to lighten the notation, we will make a slight abuse of language by using $v, l$, and $p$ to mean either point, line, and plane in $\mathbb{P}^{3}=\mathbb{P}(E)$ or their corresponding spanning vector subspace in $E=\mathbb{R}^{4}$. The features on the platform can be encoded in a flag $C_{\bullet}=\left\{0 \subset v^{*} \subset l^{*} \subset p^{*} \subset E\right\}$, i.e., a sequence of nested subspaces of each dimension, and the features on the base in a point $Z=v$ and a partial flag $Y_{\bullet}=\{0 \subset l \subset p \subset E\}$, i.e., a sequence of less than four nested subspaces.

As derived in the preceding section, the singularities of this manipulator can be characterized using its features: a configuration is singular if and only if its features satisfy $v^{*} \subset p$, $l \cap l^{*} \neq 0$, or $v \subset p^{*}$. These conditions will be compactly expressed as $v^{*}-p, l \cdot l^{*}$, and $v-p^{*}$, and they correspond to different incidences between the flag $C_{\bullet}$ and the pair point and partial flag $\left(Z, Y_{\bullet}\right)$. In order to study the structure of the singularities of the manipulator, we will construct a stratification of the configuration space of the manipulator coherent with these incidences. A stratification of a subset $S$ of a smooth manifold $M$ is a finite partition $S=\cup_{i \in I} S_{i}$ such that the components $S_{i}$, called strata, are smooth submanifolds of $M$ and satisfy a "regularity" condition, which guarantees that there are not "exceptional" degenerations between strata: if $S_{i} \cap \bar{S}_{j} \neq \emptyset$, then $S_{i} \subset \bar{S}_{j}$, where $\bar{S}_{j}$ stands for the closure of $S_{j}$. As we will see, the higher dimensional strata will correspond to the regions of nonsingular configurations, while the rest of the strata will comprise all the singularities of the manipulator. The "regularity" condition could be interpreted as follows: if in $S_{i}$ there is some singular configuration, which, after perturbation, results in entering $S_{j}$ (possibly nonsingular), then this is true for every configuration in $S_{i}$.

The construction of the stratification of the configuration space $\mathbb{R}^{3} \times \mathrm{SO}(3)$ will be performed in three steps. Since the features on the base are fixed, the partial flag $Y_{\bullet}$ and the point $Z$ remain unchanged; hence, to each manipulator configuration, we assign its corresponding flag $C_{\text {. }}$. This leads us to construct first a stratification of the flag manifold $\mathcal{F}(4)$, the variety parameterizing the spatial projective real flags, and to establish the adjacency relations between the strata (by means of an order, which will be called Bruhat order, due to the analogy to the classical one). Since the manipulator moves in the affine space $\mathbb{R}^{3}$ embedded naturally in $\mathbb{P}^{3}$, not all the spatial projective real flags are relevant to our purpose; thus, second, we will restrict the stratification found for $\mathcal{F}(4)$ to the set $\mathcal{F}_{\mathcal{A}}(4)$ of the spatial affine real flags (those whose point lies in the affine part $\mathbb{R}^{3}$ ) and prove that the "regularity" condition still holds [thus giving a stratification of $\mathcal{F}_{\mathcal{A}}(4)$ ]. Finally, we will show how the desired stratification of the configuration space $\mathbb{R}^{3} \times \mathrm{SO}(3)$ is induced via a fourfold covering morphism from $\mathcal{F}_{\mathcal{A}}(4)$. At the end, we will obtain a diagram (see Fig. 7) with the higher dimensional (5 and 6) strata and their adjacency, which completely describes the structure of the singularities of the partially flagged manipulators.

Although the steps of the reasoning are analogous to those developed in [13] and [23], it is worth noting that the 


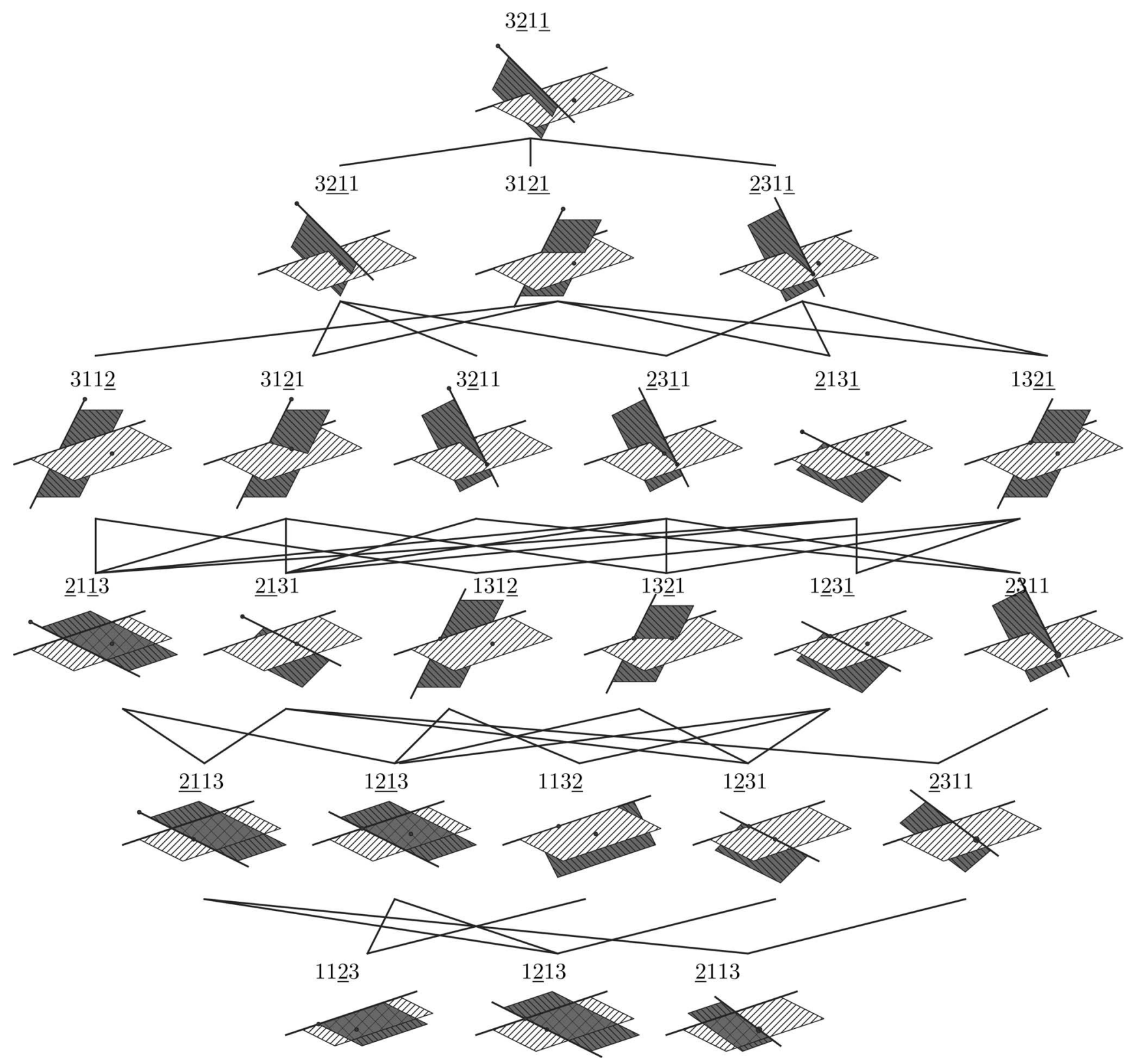

Fig. 2. Stratification derived in Theorem 1, showing the incidences between the complete flag $C_{\bullet}$ (shaded) and the reference frame formed by the point $Z$ and the partial flag $Y_{\bullet}$ (not shaded) for each stratum. The adjacency graph between strata is the Hasse diagram for the Bruhat order of decorated permutations of 1123 , whose first decorated element is $2.3 \underline{2} 1 \underline{1}$ corresponds to the orbit of dimension 6 , and $11 \underline{2} 3,1 \underline{2} 13$, and $\underline{2} 113$ characterize 1-D orbits.

stratification obtained here is formed from completely different regions, and this difference already begins at the level of the flag manifold: Compare the diagram in [23, Fig. 4] with our diagram in Fig. 2; in the latter, no zero-dimensional stratum appears, for instance. Furthermore, while the strata of $\mathcal{F}(4)$ obtained in [13] or [23] were topological cells and all together formed a cell decomposition of $\mathcal{F}(4)$, some of the strata obtained here for $\mathcal{F}(4)$ are not even cells, i.e., they are not homeomorphic to $\mathbb{R}^{n}$ (see [24]). However, it is worth remarking that both stratifications share the same structure in the higher dimensional strata, i.e., the adjacency diagram of the 5-D and 6-D strata is the same (compare [13, Fig. 16] and our Fig. 7). In fact, we venture to conjecture that this structure will be shared by all trilaterable 6-SPS parallel manipulators.

\section{A. Stratification of the Flag Manifold}

It is classically known (see [25]-[27]) that the complex flag manifolds admit a family of stratifications (in fact cell decompositions) into strata known as Bruhat or Schubert cells, given by all the possible relative positions under linear transformations between a flag and a reference flag. These stratifications are adapted in [13] to the real case and are used in the study of the singularity loci of a class of parallel manipulators, namely flagged manipulators, whose singularities can be expressed as incidences between a (complete) flag attached to the base of the manipulator $X \bullet=\{0 \subset v \subset l \subset p \subset E\}$ and another (complete) flag attached to the platform of the manipulator $C_{\bullet}=\left\{0 \subset v^{*} \subset l^{*} \subset p^{*} \subset E\right\}$. 
In this section, we will construct an alternative stratification for the real flag manifold in $E=\mathbb{R}^{4}, \mathcal{F}(4)$. In our case, each stratum of $\mathcal{F}(4)$ will comprise all the (complete) flags with the same relative position (under linear transformations) to a reference frame, which will consist of, instead of another flag, a point $v=\left\langle v_{1}\right\rangle$ and a line $l=\left\langle v_{2}, v_{3}\right\rangle$ not going through the point. Since the three generating vectors are linearly independent, there is an unique plane $p=\left\langle v_{1}, v_{2}, v_{3}\right\rangle$ that contains both the line and the point. This reference frame $(v, l, p)$ can be thought of as a partial flag, comprising the line and the plane, $Y_{\bullet}=\{0 \subset l \subset p \subset E\}$, and a point $Z=v$, with the additional property that $v \subset p$ and $v \nsubseteq l$.

We will consider that two flags have the same relative position (relative to the reference frame), i.e., they are in the same stratum, if there exists a linear transformation which, preserving the reference frame, sends one flag to the other. This describes the strata as the orbits of the action on the flag manifold $\mathcal{F}(4)$ of the subgroup $G \subset G L_{4}(\mathbb{R})$ that preserves the reference frame. Such linear transformations are used because, in this case, their orbits represent correctly the set of nonsingular configurations and the three singularity sets generated by the three trilaterations.

Let $G$ be the subgroup of $G L_{4}(\mathbb{R})$ formed by the elements that preserve the reference frame $(v, l, p)$, i.e., $G=$ $\left\{\mathbf{g} \in G L_{4}(\mathbb{R}) \mid \mathbf{g}(v)=v, \mathbf{g}(l)=l\right\}$ (the condition $\mathbf{g}(p)=p$ is a consequence of the other two conditions). Let $\Sigma$ be the permutations of the set of indexes $\{1,1,2,3\}$, i.e., $\omega \in \Sigma$ is an application $\omega:\{1,2,3,4\} \mapsto\{1,2,3\}$ that sends two elements to 1 , one element to 2 , and another to 3 ; $\omega$ will be represented by the sequence $\omega(1) \omega(2) \omega(3) \omega(4)$. A decorated permutation $(\omega, \Delta)$ is a permutation $\omega \in \Sigma$ endowed with a decoration $\Delta$, which is a nonempty sequence $j_{1}<\cdots<j_{t}$ satisfying $\omega\left(j_{1}\right)>\cdots>\omega\left(j_{t}\right) ;(\omega, \Delta)$ will be represented by the sequence $\omega(1) \omega(2) \omega(3) \omega(4)$ with the element $w(j)$ underlined (and it will be said that $w(j)$ is decorated) if, and only if, $j \in \Delta$. Observe that, from this definition, the decorated elements in the sequence $\omega(1) \omega(2) \omega(3) \omega(4)$ must appear in decreasing order.

Example: $(\omega, \Delta)=1321$ and $\left(\omega, \Delta^{\prime}\right)=1 \underline{3} 2 \underline{1}$ are decorations of the permutation $\omega=1321$, while $\underline{1} 32 \underline{1}$ and $\underline{1} 3 \underline{2} 1$ are not.

The strata of our stratification will correspond to a family of decorated permutations, which codify the incidence relations between the flag attached to the platform of the manipulator and the reference frame (partial flag and point) attached to the base (see Fig. 2).

Theorem 1: The orbits of the action of $G$ on $\mathcal{F}(4)$ are parameterized by decorated permutations $(\omega, \Delta)$ with $\omega \in \Sigma$ and whose greatest decorated element is 2 . These orbits give a stratification of $\mathcal{F}(4)$ into 24 connected strata.

An outline of the proof of this result is given in Appendix A. Our strata will be denoted by $\mathcal{O}_{(\omega, \Delta)}$, and, to lighten notations, we will sometimes use $i=(\omega, \Delta) \in I$, where $I$ stands for the set of decorated permutations of 1123 whose first decorated element is 2. Switching to the earlier examples of decorated permutations, and according to Theorem 1, 1321 corresponds to a stratum, while $1 \underline{3} 2 \underline{1}$ does not. Fig. 3(a) and (b) illustrates a configuration in the 5-D stratum $\mathcal{O}_{3211}$ and another in the 4-D stratum $\mathcal{O}_{1321}$, respectively, and gives an intuitive idea of their dimension.

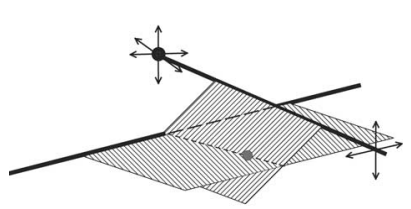

(a)

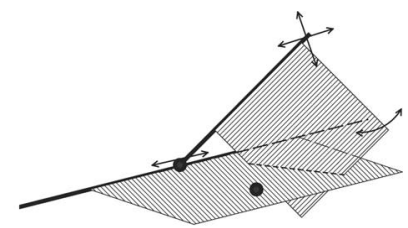

(b)
Fig. 3. (a) Configuration belonging to $\mathcal{O}_{3211}$. (b) Configuration belonging to $\mathcal{O}_{1321}$. Each double arrow represents a DOF.

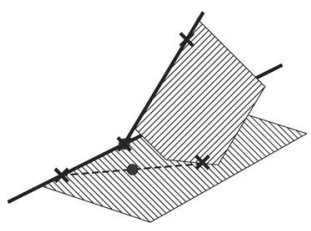

Fig. 4. Construction of a configuration in $\mathcal{O}_{1321}$.

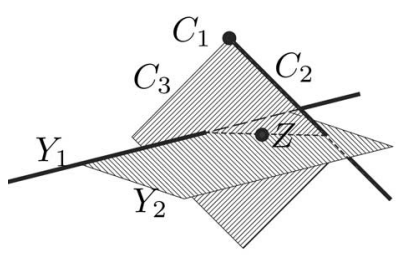

(a)

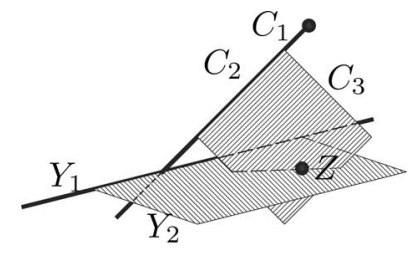

(b)
Fig. 5. Configuration in (a), belonging to the 5-D stratum $\mathcal{O}_{3211}$, degenerates to the configuration in (b), belonging to the 4-D stratum $\mathcal{O}_{312}$, by moving the line $C_{2}$ until it touches the line $Y_{1}$.

Let us see how the decorated permutation, e.g., $(\omega, \Delta)=$ 1321 , relates to its associated configurations; the partial flag $Y$. is fixed, and then, the first subspace of a possible complete flag (point) is generated with a point of the first $[\omega(1)=1]$ subspace of the partial flag (line). The second subspace (line) is generated adding a point of the third $[\omega(2)=3]$ subspace of the partial flag (volume). The third and fourth subspaces (plane, volume) are generated by adding points of the second and first $(\omega(3)=2$ and $\omega(4)=1$ ) subspaces of the partial flag (plane and line). Finally, the point $Z$ is placed in the sum of the third and fourth points we took (since the second and third elements are underlined). The configuration obtained in this way belongs to $\mathcal{O}_{1321}$. This construction can be seen in Fig. 4.

In analogy to the classical Ehresmann-Bruhat order for the permutations of four elements, a partial order for elements of $I$ can also be defined (see Appendix B), which will be called adjacency (or Bruhat) order, and it characterizes the adjacency relations between the strata of our stratification as follows:

$$
(\omega, \Delta) \leq\left(\omega^{\prime}, \Delta^{\prime}\right) \Leftrightarrow \mathcal{O}_{(\omega, \Delta)} \subseteq \overline{\mathcal{O}}_{\left(\omega^{\prime}, \Delta^{\prime}\right)} .
$$

Fig. 5 illustrates the degeneration of the 5-D stratum $\mathcal{O}_{3211}$ into the 4-D stratum $\mathcal{O}_{31 \underline{1} 1}$. In this case, $31 \underline{21} \leq 3 \underline{211}$, which means $\mathcal{O}_{3121} \subseteq \overline{\mathcal{O}}_{3211}$.

The adjacency relations between the strata can be described by drawing the Hasse diagram of the Bruhat order (Fig. 2). In a Hasse diagram of a partial order, there is an edge between two elements $a$ and $b$ if $a$ is over $b$, which are consecutive, i.e., $a>b$ 
and there is no $c$ such that $a>c>b$. In the figure, the strata are arranged in layers according to their dimension, from (top) the 6-D one down to (bottom) the 1-D ones.

\section{B. From Projective Flags to Affine Flags}

The affine space $\mathbb{R}^{3}$ can be viewed as a subspace of $\mathbb{P}^{3}$ via $\mathbb{R}^{3} \cong \mathbb{P}^{3} \backslash \Pi_{\infty}$, where $\Pi_{\infty}$ stands for the plane at infinity. In what follows, we will make a slight abuse of language by identifying the subspaces (that are affine, i.e., that are not contained in $\Pi_{\infty}$ ) of $\mathbb{P}^{3}$ with their restrictions to $\mathbb{R}^{3}$. A projective flag whose first subspace is affine, i.e., whose point is in $\mathbb{R}^{3}$, is called an affine flag, and the subset of $\mathcal{F}(4)$ parameterizing the affine flags will be denoted by $\mathcal{F}_{\mathcal{A}}(4)$. For our purpose, since we associate a flag to each configuration of the platform of a parallel manipulator, we are only interested in affine flags. For this reason, we will restrict the stratification of $\mathcal{F}(4)$ obtained previously to a partition of the subset $\mathcal{F}_{\mathcal{A}}(4)$ of affine flags and show that it still gives a stratification.

Given a stratification of the flag manifold, as for instance the one obtained in Theorem $1, \mathcal{F}(4)=\bigcup_{i \in I} \mathcal{O}_{i}$, another stratification for $\mathcal{F}_{\mathcal{A}}(4)$ is easily constructed by defining the new strata as the restriction to $\mathcal{F}_{\mathcal{A}}(4)$ of the originals

$$
\mathcal{F}_{\mathcal{A}}(4)=\mathcal{F}_{\mathcal{A}}(4) \cap \bigcup_{i \in I} \mathcal{O}_{i}=\bigcup_{i \in I}\left(\mathcal{O}_{i} \cap \mathcal{F}_{\mathcal{A}}(4)\right)=\bigcup_{i \in I} \mathcal{O}_{i}^{\prime}
$$

where $\mathcal{O}_{i}^{\prime}=\mathcal{O}_{i} \cap \mathcal{F}_{\mathcal{A}}(4)$. This clearly gives a finite partition of $\mathcal{F}_{\mathcal{A}}(4)$ into submanifolds, and the conditions of stratification are easily verified (see [24]). This stratification, however, does not guarantee the strata to be connected sets, which is an important condition in the study of singularity spaces. A new stratification can be constructed by defining each connected component of the strata of $\mathcal{F}_{\mathcal{A}}(4)$ as a stratum of the new stratification: $\mathcal{O}_{i}^{\prime}=$ $\bigcup_{\varepsilon \in N_{i}} \mathcal{O}_{i}^{\varepsilon}$, where $N_{i}$ is the set of indices of the connected components of $\mathcal{O}_{i}^{\prime}$. The number of strata grows, but in our case, the number of connected components in each $\mathcal{O}_{i}^{\prime}$ is at most 2.

Theorem 2: The partition

$$
\mathcal{F}_{\mathcal{A}}(4)=\bigcup_{i \in I} \bigcup_{\varepsilon \in N_{i}} \mathcal{O}_{i}^{\varepsilon}
$$

gives a stratification of $\mathcal{F}_{\mathcal{A}}(4)$ into 39 strata. Moreover,

1) $\sharp N_{i}=1$ in the cases where $i$ corresponds to a decorated permutation either beginning with 2 and without any 1 decorated (e.g., 2131) or beginning with 1 and none of the ones is decorated (e.g., 1231).

2) $\sharp N_{i}=2$ in the cases where $i$ corresponds to a decorated permutation either beginning with 3 (e.g., 3211), or beginning with 2 and has one 1 decorated (e.g., 2131), or beginning with 1 and the second 1 is decorated (e.g., 12 31 ).

In conclusion, the strata are divided according to Table I.

Decorated permutations in 1) correspond to configurations where either $v^{*}=v$ (permutation of type $\underline{2} * 1 *$ in Table I), or $v^{*} \subset l$ (of type $1 * 1 *$ ). On the other hand, the ones in 2 ) can be interpreted as either $v^{*} \not \subset p$ (permutation of type $3 *$ in Table I), splitting according to which side of the plane $p$ the point $v^{*}$ lies, or $v^{*} \subset p$ and $v^{*} \not \subset l$ and $v^{*} \neq v$ (of type $2 * \underline{1} *$ ), splitting depending on which side of the line $l$ and inside the plane $p$ the
TABLE I

NUMBER OF STRATA OF THE STRATIFICATION OF $\mathcal{F}(4)$ AND THEIR SUBDIVISION IN $\mathcal{F}_{\mathcal{A}}(4)$

\begin{tabular}{|c|c|c|}
\hline$(\omega, \Delta)$ & Number of strata in $\mathcal{F}(4)$ & Number of strata in $\mathcal{F}_{\mathcal{A}}(4)$ \\
\hline $3 *$ & 6 & 12 \\
$2 * 1 *$ & 3 & 3 \\
$2 * \underline{1} *$ & 6 & 12 \\
$1 * 1 *$ & 6 & 6 \\
$1 * \underline{1} *$ & 3 & 6 \\
\hline Total & 24 & 39 \\
\hline
\end{tabular}

The asterisk denotes a subset (may be empty) of nonunderlined subindexes that complete the decorated permutation (except for number 2 , which is always underlined).

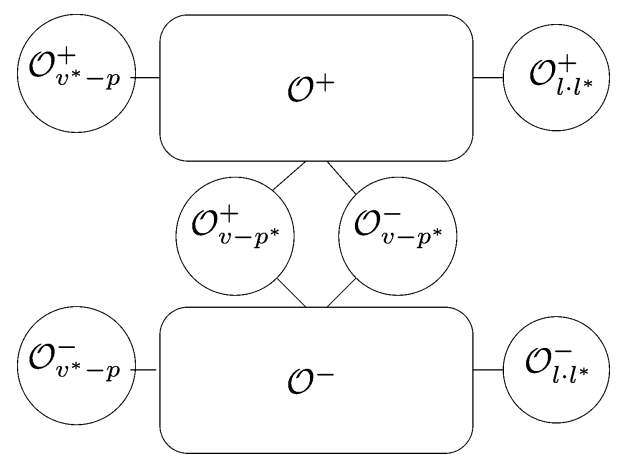

Fig. 6. Strata of dimensions 6 and 5 of the stratification of $\mathcal{F}_{\mathcal{A}}(4)$ and their adjacency relations.

point $v^{*}$ lies, or $v^{*} \subset l$ and $v \not \subset p^{*}$ (of type $1 * 1 *$, except for 1213), splitting depending on which side of $p^{*}$ the point $v$ lies, or $v \subset p^{*}$ and $v \not \subset l^{*}$ and $v^{*} \neq v$ (permutation 1213), splitting depending on which side of the line $l^{*}$ and inside the plane $p^{*}$ the point $v$ lies.

Additionally, the splitting of the strata can also be interpreted as the sign of the volume of some of the tetrahedra defining the trilateration.

In particular, the strata of dimensions 6 and 5 split each one into two connected components, and their adjacency relations are represented in Fig. 6 (see Appendix $\mathrm{C}$ for details). The rounded rectangles represent the two 6-D strata $\mathcal{O}^{+}$and $\mathcal{O}^{-}$ (connected components of $\mathcal{O}_{3 \underline{2} 1 \underline{1}}^{\prime}$ ), while the circles are the six 5-D strata: $\mathcal{O}_{v^{*}-p}^{+}$and $\mathcal{O}_{v^{*}-p}^{-}$(connected components of $\left.\mathcal{O}_{\underline{2} 31}^{\prime}\right)$, $\mathcal{O}_{l \cdot l^{*}}^{+}$and $\mathcal{O}_{l \cdot l^{*}}^{-}$(connected components of $\left.\mathcal{O}_{31 \underline{21}}^{\prime}\right)$, and $\mathcal{O}_{v-p^{*}}^{+}$ and $\mathcal{O}_{v-p^{*}}^{-}\left(\right.$connected components of $\left.\mathcal{O}_{3211}^{\prime}\right)$.

\section{From Affine Flags to Manipulator Configurations: The Topology of Singularities}

The stratification of the set $\mathcal{F}_{\mathcal{A}}(4)$ that we have just obtained induces a straightforward stratification of the configuration space (C-space) of the manipulator $\mathbb{R}^{3} \times \mathrm{SO}(3)$ via a fourfold covering morphism $\pi: \mathbb{R}^{3} \times \mathrm{SO}(3) \rightarrow \mathcal{F}_{\mathcal{A}}(4)$, which is explained in detail in [13] and [23]. In fact, $\pi$ sends to any affine flag $C_{\bullet}=\left\{0 \subset v^{*} \subset l^{*} \subset p^{*} \subset E\right\} \in \mathcal{F}_{\mathcal{A}}(4)$ (attached to the platform of the manipulator) the four positive-oriented orthonormal frames centered at $v^{*}, \mathbf{q}_{i}=\left\{v^{*} ; \mathbf{e}_{1}^{i}, \mathbf{e}_{2}^{i}, \mathbf{e}_{3}^{i}\right\}$ with $1 \leq$ $i \leq 4$, spanning $C_{\bullet}$, i.e., $v^{*}+\left\langle\mathbf{e}_{1}^{i}\right\rangle=l^{*}, v^{*}+\left\langle\mathbf{e}_{1}^{i}, \mathbf{e}_{2}^{i}\right\rangle=p^{*}$. It holds that $\left\{\mathbf{q}_{i}\right\}_{1 \leq i \leq 4}=\left\{\mathbf{q}_{i_{0}}, \mathbf{R}_{1} \mathbf{q}_{i_{0}}, \mathbf{R}_{2} \mathbf{q}_{i_{0}}, \mathbf{R}_{3} \mathbf{q}_{i_{0}}\right\}$, where $\mathbf{R}_{k}$ stands for a rotation of $\pi$ rad about the axis $v^{*}+\left\langle\mathbf{e}_{k}^{i_{0}}\right\rangle$, for 


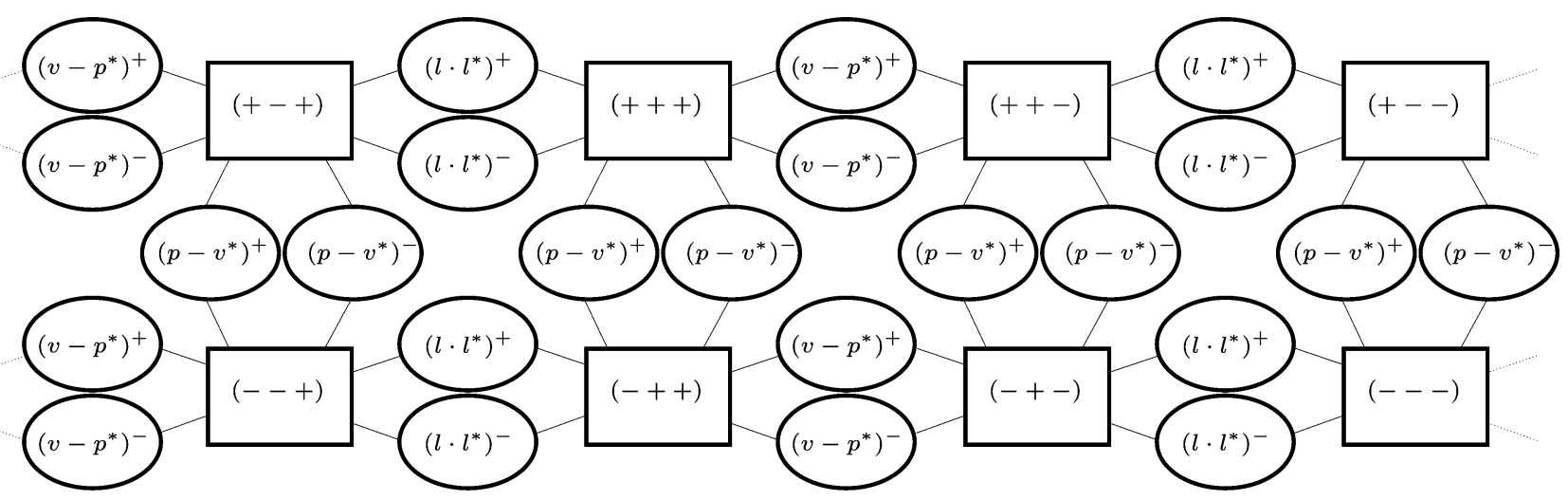

Fig. 7. Graph showing the topology of C-space for partially flagged manipulators. The rectangles represent the 6-D cells of C-space nonsingular configurations that correspond to the eight different assembly modes, while the ellipses are the 5-D cells of singular configurations.

whatever given $i_{0} \in\{1, \ldots, 4\}$. Observe that, while $C$ • gives the affine features of the relative position between base and platform, $\left\{\mathbf{q}_{i}\right\}_{1 \leq i \leq 4}$ give the four platform configurations sharing the same flag $C$.

This $\pi$ provides a procedure to pull back the stratification of the affine flags to obtain a useful decomposition of the C-space of the manipulator, since the singularity locus corresponds to the strata of dimension lower than or equal to 5 . Hence, $\mathbb{R}^{3} \times \mathrm{SO}(3)$ is divided into eight 6-D strata [four times two 6-D disjoint connected strata in $\mathcal{F}_{\mathcal{A}}(4)$ ], which, by connectedness arguments (since $\mathcal{F}_{\mathcal{A}}(4)$ is path-connected), are eight connected components of the nonsingular configurations corresponding to the eight assembly modes of the manipulator. They are separated by 24 5-D strata of singular configurations [four times six 5-D disjoint strata in $\mathcal{F}_{\mathcal{A}}(4)$ ]. Analogously, as in [13], by resorting to the theory of path lifting [28], the adjacencies between these eight 6-D strata and 24 5-D strata can be derived, resulting in the graph shown in Fig. 7. The rectangles represent the eight 6-D connected components of C-space of nonsingular configurations, while the ellipses are the 5-D manifold patches of singular configurations separating these components. As noted before, it is remarkable that at this level the adjacency diagram is the same as the one obtained in [13] for the flagged manipulators, despite the stratification obtained here being formed from different regions.

In Fig. 7, to characterize each 6-D stratum, we use a triple of signs corresponding to the orientation of the three tetrahedra appearing in the trilaterations, which solve the forward kinematics of the manipulator. Each of the four 5-D strata coming from $\mathcal{O}_{i}^{\varepsilon} \in \mathcal{F}_{\mathcal{A}}(4)$ is denoted by $(i)^{\varepsilon}$ (as, for instance, the four strata $\left(v-p^{*}\right)^{+}$mapping to $\mathcal{O}_{v-p^{*}}^{+}$by $\left.\pi\right)$.

\section{Singularity-Free REDUNDANT MANIPULATOR}

In this section, we study all the ways in which an extra leg can be added to a 3-3 partially flagged manipulator and analyze their effect on singularities.

The only actuators that can be added to the partially flagged 3-3 manipulator shown in Fig. 1(c) are $B Z, C X$, and $C Y$. Since the results obtained by adding $C X$ or adding $C Y$ are equivalent due to the symmetry of $X$ and $Y$, we will only consider the
TABLE II

List of Possible MANipulators ObTained by AdDing THE ACTUATOR $B Z$ TO THE ORIGINAL ONE

\begin{tabular}{|c|c|c|c|}
\hline Add & Remove & Features & Comments \\
\hline$B Z$ & $A X$ & $\begin{array}{l}(Z, Y Z, X Y Z \\
B, A B, A B C)\end{array}$ & a flagged manipulator ${ }^{\dagger}$ \\
\hline$B Z$ & $A Y$ & $\begin{array}{l}(Z, X Z, X Y Z \\
B, A B, A B C)\end{array}$ & a flagged manipulator ${ }^{\dagger}$ \\
\hline$B Z$ & $A Z$ & $\begin{array}{l}(Z, X Y, X Y Z \\
B, A B, A B C)\end{array}$ & $\begin{array}{l}\text { a partially-flagged } \\
\text { manipulator }{ }^{\dagger \ddagger}\end{array}$ \\
\hline$B Z$ & $B X$ & $\begin{array}{l}(Z, Y Z, X Y Z \\
A, A B, A B C)\end{array}$ & a flagged manipulator ${ }^{\dagger \S}$ \\
\hline$B Z$ & $B Y$ & $\begin{array}{l}(Z, X Z, X Y Z \\
A, A B, A B C)\end{array}$ & a flagged manipulator ${ }^{\dagger \S}$ \\
\hline$B Z$ & $C Z$ & - & architecturally singular \\
\hline
\end{tabular}

TABLE III

List OF Possible MANiPUlators OBTAINED By AdDING THE ACTUATOR $C Y$ TO THE ORIGINAL ONE

\begin{tabular}{|c|c|c|c|}
\hline Add & Remove & Features & Comments \\
\hline$C Y$ & $A X$ & $\begin{array}{l}(Y, Y Z, X Y Z \\
B, A C, A B C)\end{array}$ & $\begin{array}{l}\text { an inverted partially- } \\
\text { flagged manipulator }\end{array}$ \\
\hline$C Y$ & $A Y$ & - & $\begin{array}{l}\text { an octahedral manipulator } \\
(2-2-2)\end{array}$ \\
\hline$C Y$ & $A Z$ & $\begin{array}{l}(Y, X Y, X Y Z \\
C, A B, A B C)\end{array}$ & $\begin{array}{l}\text { an inverted partially- } \\
\text { flagged manipulator }\end{array}$ \\
\hline$C Y$ & $B X$ & $\begin{array}{l}(Y, Y Z, X Y Z \\
A, A C, A B C)\end{array}$ & a flagged manipulator ${ }^{\S}$ \\
\hline$C Y$ & $B Y$ & $\begin{array}{l}(X, Y Z, X Y Z \\
A, A C, A B C)\end{array}$ & $\begin{array}{l}\text { a partially-flagged } \\
\text { manipulator }^{\S}\end{array}$ \\
\hline$C Y$ & $C Z$ & $\begin{array}{l}(Y, X Y, X Y Z \\
A, A B, A B C)\end{array}$ & A flagged manipulator ${ }^{\ddagger \S}$ \\
\hline
\end{tabular}

cases $B Z$ and $C Y$. In Tables II and III, we see all the possible combinations of enabling and disabling a leg, and the features shared with the original manipulator are also indicated. The combinations sharing the singularity corresponding to $v-p^{*}$ are marked with ${ }^{\dagger}, l \cdot l^{*}$ with ${ }^{\ddagger}$, and $v^{*}-p$ with ${ }^{\S}$.

All the possible manipulators obtained after adding the actuator $B Z$ (Table II) preserve the 5-D singularity $v-p^{*}$, $Z \in A B C$, and therefore, it will be present in the singularity loci of the redundant manipulator. Their nonsingular configuration space will consist of two 6-D connected components separated by a 5 -D singularity. 
TABLE IV

INTERSECTIONS OF THE SINGULARITIES OF TWO OF THE MANIPULATORS PRESENT IN THE STUDIED 7-LEGGED STRUCTURE

\begin{tabular}{|c||c|c|c|}
\hline & $v_{1}-p_{1}^{\prime}$ & $l_{1} \cdot l_{1}^{\prime}$ & $v_{1}^{\prime}-p_{1}$ \\
\hline$v_{2}-p_{2}^{\prime}$ & $Z \in A B C$ & $X Y \cap A B \neq \emptyset$ & $A \in X Y Z$ \\
$Y \in A B C$ & $Y Z \subseteq A B C$ & $(Y \in A B) \cup$ & $A Y \subseteq(A B C \cap$ \\
\hline$l_{2} \cdot l_{2}^{\prime}$ & $(Z \in A C) \cup$ & $\left(l_{1} \cdot l_{1}^{\prime}\right) \cap$ & $(A \in X Y)$ \\
$Y Z \cap A C \neq \emptyset$ & $(Y Z \subseteq A B C)$ & $\left(l_{2} \cdot l_{2}^{\prime}\right)$ & $(A C \subseteq X Y Z)$ \\
\hline$v_{2}^{\prime}-p_{2}$ & $B Z \subseteq(A B C \cap$ & $(B \in X Y) \cup$ & \multirow{2}{*}{$A B \subseteq X Y Z$} \\
$B \in X Y Z$ & $X Y Z)$ & $(A B \subseteq X Y Z)$ & \\
\hline
\end{tabular}

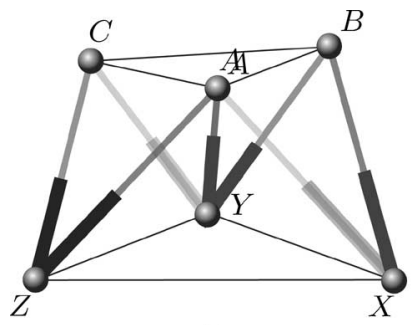

(a)

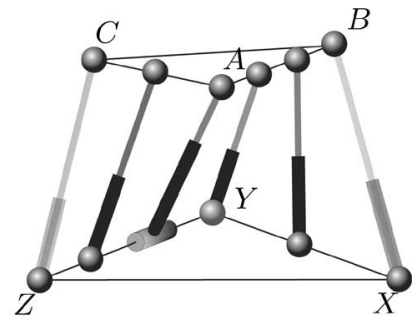

(b)
Fig. 8. (a) Redundant 3-3 parallel manipulator. By enabling one of the actuators shown in light grey while disabling the other, the manipulator can exchange its structure between a basic partially flagged manipulator (enabling $A X$ ) and an inverted basic partially flagged manipulator (enabling $C Y$ ). (b) Redundant parallel manipulator without multiple spherical joints that has the same singularity locus as that in (a).

When adding the actuator $C Y$ (or, equivalently, $C X$ ) the results are richer (see Table III). In this case, there is a combination without any shared 5-D singularity stratum with the original manipulator. A manipulator with actuators $A X, A Y$, $A Z, B X, B Y, C Y$, and $C Z$, disabling $A X$ or $C Y$, has a connected nonsingular configuration space and its singularity locus has dimension 4 . This can be seen by computing the dimension (DOFs) of the nine intersections of the singularity loci, as shown in Table IV, straightforward for all the cases, except for $\left(l_{1} \cdot l_{1}^{\prime}\right) \cap\left(l_{2} \cdot l_{2}^{\prime}\right)$, which is more complex.

In conclusion, a redundant manipulator with triangular base and platform can be constructed [see Fig. 8(a)] having a 6-D workspace with singularity sets of dimension 4 and, hence, having a connected set of nonsingular configurations.

It is worth mentioning that the singularity loci cannot be further reduced from dimension 4 , since the set $(Y Z \cap A C \neq$ $\emptyset) \cap(X Y \cap A B \neq \emptyset)$, of dimension 4, will always be present.

As a shortcoming, one may think that the practical implementation of the resulting platform would involve a formidable design task because of the presence of multiple spherical joints. Fortunately, these joints can be eliminated by substituting the point-line components, i.e., sets of two legs sharing an attachment, with other equivalent kinematic chains without altering the singularities of the platform. The geometric conditions that must be satisfied so that a point-line component can be substituted by another point-line component without altering the singularity locus of the platform are discussed in [29]. How a point-line component can be substituted by a serial kinematic chain, in particular, a $\underline{P} R \underline{P}$ chain, without altering the parallel singularities of the platform is discussed in [30]. Using these two kinds of substitutions, it is possible to derive the design in
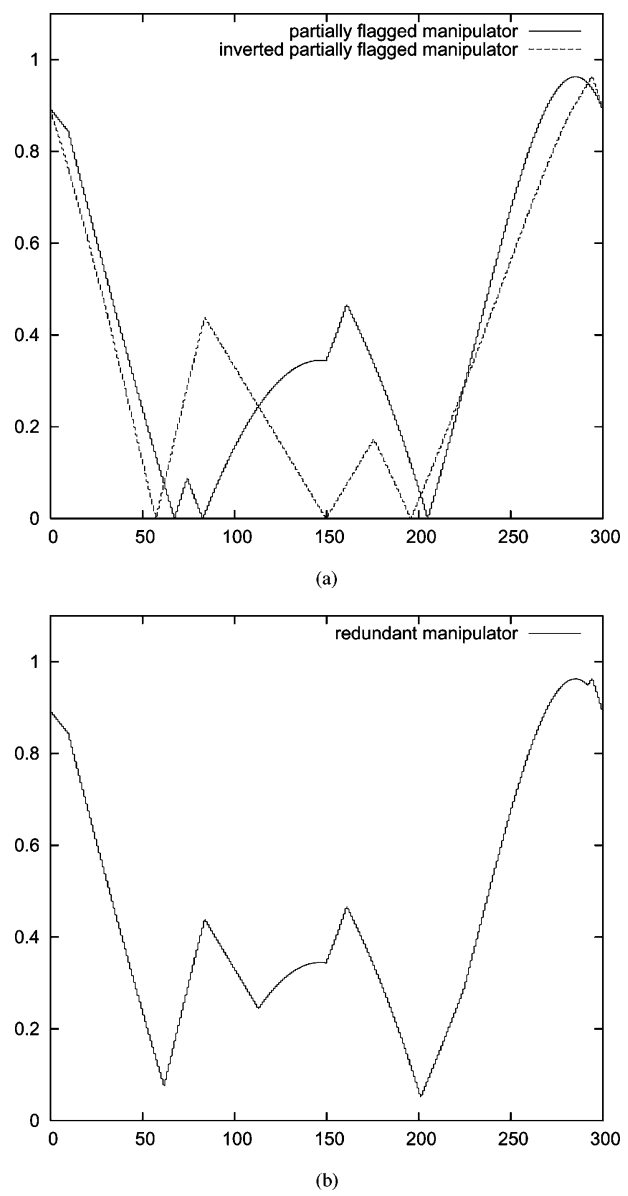

Fig. 9. Evolution of the singularity detection function $F(\mathbf{q})$ along a path for (a) the two component manipulators and (b) the redundant manipulator.

Fig. 8(b), which has the same singularity locus as that in Fig.8(a), but where all multiple spherical joints have been eliminated.

\section{Simulation Results}

The simulations have been performed using $\mathrm{C}++$ for the computation of the distances and configurations, and Blender to visualize the simulations that required control over the actuators.

As explained in Section II, the singularities of a partially flagged manipulator can be characterized by the degeneration of either one of the tetrahedra $A X Y Z, A B X Y$, or $A B C Z$. Thus, the function

$$
F(\mathbf{q})=\min \left\{\operatorname{Vol}_{A X Y Z}, \operatorname{Vol}_{A B X Y}, \operatorname{Vol}_{A B C Z}\right\}
$$

provides a measure of how far the configuration $\mathbf{q}$ is from a singularity. When applying switched control to our redundant manipulator, we use this function to determine which of the two component manipulators is farther from a singular configuration and, consequently, which actuator must be passive.

The most important advantage of our redundant manipulator is its connected singularity-free workspace. To exemplify this characteristic, we have planned a path from a configuration with the platform parallel to the base and over it, to a configuration with the platform under the base. These configurations belong to distant nonsingular 6-D strata of the two component 
TABLE V

Strata Crossed by the Two Structures of the REDUNDANT MANIPULATOR

\begin{tabular}{|c|c|c|}
\hline time & partially flagged & inverted partially flagged \\
\hline 0 & --+ & -+- \\
57 & --+ & -+- \\
58 & -++ & -+- \\
67 & -++ & -+- \\
68 & -++ & -++ \\
83 & -++ & -++ \\
84 & -++ & +++ \\
150 & -++ & +++ \\
151 & +++ & +++ \\
196 & +++ & +++ \\
197 & ++- & +++ \\
206 & ++- & +++ \\
207 & ++- & +-+ \\
300 & ++- & +-+ \\
\hline
\end{tabular}

manipulators forming the redundant one: from $\mathcal{O}^{--+}$to $\mathcal{O}^{++-}$ for the partially flagged manipulator (Fig. 8, with $A X$ active) and from $\mathcal{O}^{-+-}$to $\mathcal{O}^{+-+}$in the case of the inverted partially flagged manipulator (Fig. 8, with $C Y$ active). Hence, in both cases, the manipulator has to cross three singular 5-D strata, corresponding to $v-p^{*}, l \cdot l^{*}$, and $v^{*}-p$.

This can be seen in Fig. 9, where the evolution of the singularity detection function $F(\mathbf{q})$, i.e., the minimum of the three tetrahedra volumes, along a nonsingular path is plotted for the two component manipulators [see Fig. 9(a)] and the redundant composite one [see Fig. 9(b)]. Since in each configuration the control strategy chooses the less singular structure, the singularity detection function of the redundant manipulator is the maximum of the functions of both components. Note that this function does not nullify along the path, since there are no simultaneous singularities in the two component manipulators. The strata crossed by each component manipulator, which is labeled with the signs of the three tetrahedra, along the path are shown in Table V.

Videos of this simulation, as well as further experimental results, can be found in [24].

Note that, since the simultaneous singularities form 4-D sets in a 6-D space, any singular path can be slightly perturbed in order to obtain a nonsingular path.

\section{CONCLUSION AND Future PRosPeCts}

The configuration space (C-space) of the partially flagged 3-3 manipulator consists of eight 6-D regions (corresponding to the eight solutions of its forward kinematics) separated by singular 5 -D hypersurfaces. Building on classical results on the stratification of flag manifolds, we have derived a complete description of this $\mathrm{C}$-space in terms of disjoint regions of decreasing dimensionalities, such that a region of dimension $k$ is entirely in the boundary of a region of dimension $k+1$ and, in turn, is bounded by regions of dimension $k-1$.

This provides a detailed charting of the singular hypersurfaces consisting of all the regions of dimension 5 and lower, and thus, it is a powerful tool to avoid singularities, both at the manipulator design and path planning stages. For instance, it has been shown that, by adding a seventh leg to a partially

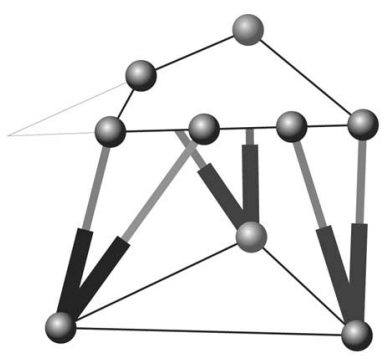

(a)

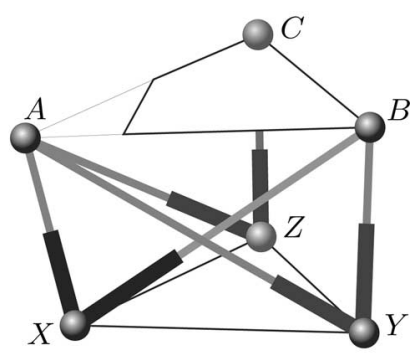

(b)
Fig. 10. (a) Partially flagged 6-3 parallel manipulator and (b) its singularityequivalent partially flagged 3-3 manipulator.

flagged manipulator and applying switching control, the eight nonsingular 6-D regions in the initial C-space are fused into just one region for the redundant manipulator, due to the removal of the singular 5-D hypersurface patches, and consequently, the manipulator workspace is considerably enlarged. Concerning path generation, the strategy used here for illustrative purposes (straight path and, if necessary, perturbation) is the simplest possible that avoids singularities locally. More complex control algorithms are being developed for global path optimization based on the graph of strata and subject to two types of constraints: avoiding interferences between the platform, the base, and the legs and keeping the actuators within their operational ranges.

It is worth noting that the obtained C-space decomposition is valid for the whole family of partially flagged manipulators, i.e., those sharing the same singular hypersurfaces. Although the derivation of this family is beyond the scope of this paper, let us provide a glimpse of how it would develop. By applying successive kinematics-preserving transforms [29] to subsets of legs of the partially flagged 3-3 manipulator, other kinematically equivalent designs are obtained. Fig. 10 provides an example, where a partially flagged 6-3 manipulator is shown to derive from the 3-3 one. Let us mention that this separation of multiple spherical joints is most useful from the practical design viewpoint.

A similar singularity charting had previously been attained for flagged manipulators [13], [23], which, remarkably, had the same structure for 5-D and 6-D strata. However, there are important differences between the two decompositions, the most interesting from our viewpoint being that the 0 -D strata in the C-space of flagged manipulators do not exist for partially flagged ones. This is due to the fact that a complete flag has degenerated into a partial one, because the vertex is no longer contained in the corresponding line. It looks as if the disintegration of the flag would lead to the progressive disappearance of low-dimensional strata until reaching the limit of 3-D strata, which must necessarily be present because some of them depend on individual flag elements. Therefore, we plan to study the consequences of taking the vertex out of the plane by using the results of [31] on two subspaces and a flag and, afterward, those of taking the line out of the plane. Our conjecture is that the same graph of 5-D and 6-D strata (see Fig. 7) will be shared by all trilaterable 6-SPS parallel manipulators. 


\section{APPENDIX A}

\section{StRATIFICATION OF THE FlaG MANIFOLd $\mathcal{F}(4)$}

The aim of this part of the Appendix is to present the reasonings that lead to prove Theorem 1, which gives a stratification of the flag manifold $\mathcal{F}(4)$ suitable for our practical purpose: to provide a complete description of the singularities of the class of parallel robots named as partially flagged manipulators. First of all, we will study the stratification of all the possible configurations between a point and two flags and use this information to obtain the desired stratification of $\mathcal{F}(4)$.

Keeping the notations of Section III (see also Table VI), let $\mathcal{F}(\mathbf{b})$ be the variety parameterizing the partial flags of the type $Y_{\bullet}=\left\{0 \subset l \subset p \subset E=\mathbb{R}^{4}\right\}$, where $l$ and $p$ span a line and plane, respectively, in $\mathbb{P}^{3}=\mathbb{P}(E)$. Consider the variety $\mathbb{P}^{3} \times \mathcal{F}(\mathbf{b}) \times \mathcal{F}(4)$, which describes all possible configurations of a point $Z$ in $\mathbb{P}^{3}$, a partial flag $Y_{\bullet} \in \mathcal{F}(\mathbf{b})$, and a flag $C_{\bullet} \in \mathcal{F}(4)$, and let the linear group $G L_{4}(\mathbb{R})$ act diagonally on $\mathbb{P}^{3} \times \mathcal{F}(\mathbf{b}) \times \mathcal{F}(4)$, i.e., $\mathbf{g}\left(Z, Y_{\bullet}, C_{\bullet}\right)=\left(\mathbf{g}(Z), \mathbf{g}\left(Y_{\bullet}\right), \mathbf{g}\left(C_{\bullet}\right)\right)$ for any $g \in G L_{4}(\mathbb{R})$. This action is studied by Magyar [32], and his results can be summarized as follows (adapted over $\mathbb{R}$, which is the case that concerns us).

Theorem 3 ([32]):

1) The orbits of the action of $G L_{4}(\mathbb{R})$ on $\mathbb{P}^{3} \times \mathcal{F}(\mathbf{b}) \times \mathcal{F}(4)$ are parameterized by decorated permutations of 1123 , and there are 82 different orbits $\mathcal{D}_{(\omega, \Delta)}$ of this action.

2) A partial order $\stackrel{\text { deg }}{\leq}$ (named degeneration order) can be defined that characterizes the degeneration in Zariski topology between the orbits

$$
(\omega, \Delta) \stackrel{\operatorname{deg}}{\leq}\left(\omega^{\prime}, \Delta^{\prime}\right) \Leftrightarrow \mathcal{D}_{(\omega, \Delta)} \cap \overline{\mathcal{D}}_{\left(\omega^{\prime}, \Delta^{\prime}\right)} \neq \emptyset .
$$

It is shown in [24] that this action, in fact, gives a stratification of $\mathbb{P}^{3} \times \mathcal{F}(\mathbf{b}) \times \mathcal{F}(4)$ and that the degeneration order also characterizes degeneration between orbits in Euclidean topology (over $\mathbb{R}$ ) and, furthermore, the adjacency between orbits

$$
(\omega, \Delta) \stackrel{\operatorname{deg}}{\leq}\left(\omega^{\prime}, \Delta^{\prime}\right) \Leftrightarrow \mathcal{D}_{(\omega, \Delta)} \subseteq \overline{\mathcal{D}}_{\left(\omega^{\prime}, \Delta^{\prime}\right)}
$$

Once the structure of the orbits of the action of $G L_{4}(\mathbb{R})$ on $\mathbb{P}^{3} \times \mathcal{F}(\mathbf{b}) \times \mathcal{F}(4)$ has been established, the stratification of $\mathcal{F}(4)$ can be deduced. We will show that the orbits of the action of $G$ on $\mathcal{F}(4)$, which are the strata of our stratification (see Section III-A), correspond to subsets of the orbits of $G L_{4}(\mathbb{R})$ on $\mathbb{P}^{3} \times \mathcal{F}(\mathbf{b}) \times \mathcal{F}(4)$.

Recall that, for our practical purpose, we have a reference frame $(v, l, p)$ attached to the base of the robot consisting of a point $v$, a line $l$, and a plane $p$ satisfying $v \nsubseteq l \subset p$ and $v \subset p$, which can be thought as a partial flag $Y_{\bullet}=\{0 \subset l \subset$ $p \subset E\} \in \mathcal{F}(\mathbf{b})$ and a point $Z=v \in \mathbb{P}^{3}$, and that we have a flag $C_{\bullet}=\left\{0 \subset v^{*} \subset l^{*} \subset p^{*} \subset E\right\} \in \mathcal{F}(4)$ attached to the platform. Observe that, in our application, the pair $\left(Z, Y_{\bullet}\right)$ remains fixed (and depends on each robot instance) whereas $C$. varies as the robot moves: we are only interested in the relative position between $C_{\bullet}$ and the reference frame $\left(Z, Y_{\bullet}\right)$. It is worth noting that the topological results stated in this paper are valid, irrespective of the choice of $\left(Z, Y_{\bullet}\right)$, provided
TABLE VI

\begin{tabular}{|c|c|}
\hline Symbol & Explanation \\
\hline$d_{1} \ldots d_{6}$ & Lengths of the 6 actuators of a 3-3 manipulator. \\
\hline$A B C, X Y Z$ & $\begin{array}{l}\text { Joints of the manipulator in the platform and } \\
\text { base, respectively. }\end{array}$ \\
\hline$(x, y, z),\left(\theta_{x}, \theta_{y}, \theta_{z}\right)$ & Translation and rotation of the platform centered at $A$. \\
\hline$v, l, p$ & $\begin{array}{l}\text { Point, line and plane of the base corresponding } \\
\text { to } Z, X Y, X Y Z \text {. }\end{array}$ \\
\hline$v^{*}, l^{*}, p^{*}$ & $\begin{array}{l}\text { Point, line and plane of the platform, corresponding } \\
\text { to } A, A B, A B C \text {. }\end{array}$ \\
\hline$E$ & $\mathbb{R}^{4}$ \\
\hline $\mathbb{P}^{3}=\mathbb{P}\left(\mathbb{R}^{4}\right)$ & Projective space. \\
\hline$\Pi_{\infty}$ & Plane at infinity. \\
\hline $\mathbb{R}^{3} \cong \mathbb{P}^{3} \backslash \Pi_{\infty}$ & Affine space embedded in $\mathbb{P}^{3}$. \\
\hline$C$ & $\begin{array}{l}\text { Flag }\left\{0 \subset v^{*} \subset l^{*} \subset p^{*} \subset E\right\}=\{0= \\
\left.C_{0} \subset C_{1} \subset C_{2} \subset C_{3} \subset C_{4}=E\right\}\end{array}$ \\
\hline$Y_{\bullet}$ & $\begin{array}{l}\text { Partial flag }\{0 \subset l \subset p \subset E\}=\{0= \\
\left.Y_{0} \subset Y_{2} \subset Y_{3} \subset Y_{4}=E\right\}\end{array}$ \\
\hline$\left(X, Y_{\bullet}\right)$ & Pair of point and partial flag. \\
\hline$v^{*}-p$ & Point $v^{*}$ is contained in $p$. \\
\hline$l \cdot l^{*}$ & Lines $l$ and $l^{*}$ intersect. \\
\hline $\bar{S}_{j}$ & Closure of $S_{j}$ \\
\hline $\begin{array}{l}\mathbb{R}^{3} \times \underset{\mathcal{S O O}}{ }(3) \\
\mathcal{F}(4)\end{array}$ & $\begin{array}{l}\text { Configuration space of the platform. } \\
\text { Flag manifold parameterizing real flags of } \mathbb{P}^{3} \text {. }\end{array}$ \\
\hline $\mathcal{F}_{\mathcal{A}}(4)$ & $\begin{array}{l}\text { Subset of } \mathcal{F}(4) \text { formed by the affine flags, } \\
\text { i.e. those whose point is in } \mathbb{R}^{3} \text {. }\end{array}$ \\
\hline $\begin{array}{c}\omega \\
(\omega, \Delta)\end{array}$ & $\begin{array}{l}\text { Permutation of the set of indices }\{1,1,2,3\} \text {. } \\
\text { Decorated permutation. }\end{array}$ \\
\hline$I$ & $\begin{array}{l}\text { Set of decorated permutations whose first } \\
\text { decorated element is } 2 \text {. }\end{array}$ \\
\hline$\leq$ & $\begin{array}{l}\text { Adjacency (or Bruhat) order between the } \\
\text { elements of } I \text {. }\end{array}$ \\
\hline$G L_{4}(\mathbb{R})$ & Linear group of $\mathbb{R}^{4}$ \\
\hline$G$ & Subgroup of $G L_{4}(\mathbb{R})$ that preserves $(v, l, p)$. \\
\hline $\mathcal{O}_{(\omega, \Delta)}=O r_{i}$ & $\begin{array}{l}\text { Stratum of } \mathcal{F}(4) \quad \text { parameterized by } \\
(\omega, \Delta)=i \in I\end{array}$ \\
\hline $\mathcal{O}_{(\omega, \Delta)}^{\prime}$ & Affine flags of $\mathcal{O}_{(\omega, \Delta)}$, i.e. $\mathcal{O}_{(\omega, \Delta)} \cap \mathcal{F}_{\mathcal{A}}(4)$. \\
\hline $\mathcal{O}_{(\omega, \Delta)}^{\epsilon}$ & Connected component of $\mathcal{O}_{(\omega, \Delta)}^{\prime}$, with $\epsilon \in\{+,-\}$. \\
\hline $\mathcal{O}^{+}, \mathcal{O}^{-}$ & Connected components of $\mathcal{O}_{3 \underline{2} 1 \underline{1}}^{\prime}$ \\
\hline $\mathcal{O}_{v^{*}-p}^{+}, \mathcal{O}_{v^{*}-p}^{-}$ & Connected components of $\mathcal{O}_{\underline{2} 31 \underline{1}}^{\prime}$. \\
\hline $\mathcal{O}^{++-}=(++-)$ & $\begin{array}{l}\text { 6D stratum of } \mathbb{R}^{3} \times \mathrm{SO}(3) \text {, coming from } \\
\mathcal{O}^{+} \text {or } \mathcal{O}^{-}\end{array}$ \\
\hline$\left(v^{*}-p\right)^{+}$ & $6 \mathrm{D}$ stratum of $\mathbb{R}^{3} \times \mathrm{SO}(3)$, coming from $\mathcal{O}_{v^{*}-p}^{+}$ \\
\hline $\mathbf{q}_{i}$ & $\begin{array}{l}\text { Positive oriented orthonormal frame centered } \\
\text { at } v^{*} \text { spanning } C_{\bullet} \text {. }\end{array}$ \\
\hline $\mathbf{q}$ & Configuration of the manipulator. \\
\hline $\mathcal{F}(\mathbf{b})$ & Manifold of partial flags of the type $Y_{\bullet}$. \\
\hline $\mathcal{D}_{(\omega, \Delta)}$ & $\begin{array}{l}\text { Orbit of } \mathbb{P}^{3} \times \mathcal{F}(\mathbf{b}) \times \mathcal{F}(4) \text { parameterized } \\
\text { by }(\omega, \Delta) \text {. }\end{array}$ \\
\hline g & Element of $G \subset G L_{4}(\mathbb{R})$ \\
\hline$\left(\mathbf{e}_{1}, \mathbf{e}_{2}, \mathbf{e}_{3}, \mathbf{e}_{4}\right)$ & Basis of E. \\
\hline$V=\left\{\mathbf{v}_{\omega(j) j}\right\}_{1 \leq i \leq 4}$ & $\begin{array}{l}\text { Basis of } \mathrm{E} \text { consisting of a reindexation of } \\
\left(\mathbf{e}_{1}, \mathbf{e}_{2}, \mathbf{e}_{3}, \mathbf{e}_{4}\right) \text { but for one vector. }\end{array}$ \\
\hline$C_{\bullet}^{(\omega, \Delta)}$ & Standard flag associated to $(\omega, \Delta)$ \\
\hline$*$ & In a representation of $C_{\bullet}$, any real number. \\
\hline$\sharp$ & In a representation of $C_{\bullet}$, any non zero real number. \\
\hline
\end{tabular}

LIST OF NOTATION

$v \nsubseteq l \subset p$ and $v \subset p$ are satisfied. This leads us to fix a pair $\left(Z, Y_{\bullet}\right)$ as before and assign to each flag $C_{\bullet} \in \mathcal{F}(4)$ the triplet $j\left(C_{\bullet}\right)=\left(Z, Y_{\bullet}, C_{\bullet}\right)$ in $\mathbb{P}^{3} \times \mathcal{F}(\mathbf{b}) \times \mathcal{F}(4)$. This assignation defines an embedding $j$ of $\mathcal{F}(4)$ in $\mathbb{P}^{3} \times \mathcal{F}(\mathbf{b}) \times \mathcal{F}(4)$, which is coherent with the actions of $G$ and $G L_{4}(\mathbb{R})$ on their respective spaces (see [24]). Two flags are $G$-equivalent, meaning that there exists an element of $G$ mapping one flag to the other, if and only if their images by $j$ are $G L_{4}(\mathbb{R})$-equivalent, i.e.,

$$
C_{\bullet} \stackrel{G}{\sim} C_{\bullet}^{\prime} \Leftrightarrow j\left(C_{\bullet}\right)=\left(Z, Y_{\bullet}, C_{\bullet}\right) \stackrel{G L_{4}(\mathbb{R})}{\sim}\left(Z, Y_{\bullet}, C_{\bullet}^{\prime}\right)=j\left(C_{\bullet}^{\prime}\right) .
$$

Since the orbits of an action are the sets of equivalent elements, two flags belong to the same orbit in $\mathcal{F}(4)$ if and only if their images by $j$ belong to the same orbit in $\mathbb{P}^{3} \times \mathcal{F}(\mathbf{b}) \times \mathcal{F}(4)$. Hence, 
each orbit in $\mathcal{F}(4)$ is injected to an orbit in $\mathbb{P}^{3} \times \mathcal{F}(\mathbf{b}) \times \mathcal{F}(4)$, and the assignation of decorated permutations to the orbits of $G$ in $\mathcal{F}(4)$ follows. Since in our application the relative position between $Z$ and $Y_{\bullet}$ is fixed, not all the strata in $\mathbb{P}^{3} \times \mathcal{F}(\mathbf{b}) \times \mathcal{F}(4)$ have a nonempty intersection with $j(\mathcal{F}(4))$. We have proved in [24] that only the strata in $\mathbb{P}^{3} \times \mathcal{F}(\mathbf{b}) \times \mathcal{F}(4)$ corresponding to a decorated permutation whose greatest decorated element is 2 contain the image of an orbit of the action of $G$ on $\mathcal{F}(4)$ (which are those relevant for us; cf., the statement of Theorem 1). When reasoning that the orbits of the action of $G$ on $\mathcal{F}(4)$ provide a stratification, the more delicate point is to verify the boundary condition. It follows that once we have shown that the restriction to $\mathcal{F}(4)$ by $j$ of the degeneration order (introduced in Theorem 3) also describes the adjacency between orbits in $\mathcal{F}(4)$ (and, for this reason, it is taken in Section III-A as the Bruhat order), we need to prove the equivalency

$$
\begin{aligned}
& \mathcal{O}_{(\omega, \Delta)} \subseteq \overline{\mathcal{O}}_{(\widetilde{\omega}, \widetilde{\Delta})} \text { in } \mathcal{F}(4) \\
& \Leftrightarrow \mathcal{D}_{(\omega, \Delta)} \subseteq \overline{\mathcal{D}}_{(\widetilde{\omega}, \widetilde{\Delta})} \text { in } \mathbb{P}^{3} \times \mathcal{F}(\mathbf{b}) \times \mathcal{F}(4) .
\end{aligned}
$$

The left-to-right implication simply follows in virtue of the continuity of $j$ and the good behavior of the boundary condition under actions of groups on manifolds (see [24]). The opposite implication is not true for a generic subset of $\mathbb{P}^{3} \times \mathcal{F}(\mathbf{b}) \times$ $\mathcal{F}(4)$, but in the case of $\mathcal{F}(4)$, we have proved it in [24] using a deeper understanding of the adjacency relations.

\section{APPENDIX B}

\section{CHARACTERIZATION OF THE STRATA OF $\mathcal{F}(4)$}

In this part of the Appendix, we will describe the flags that comprise each stratum of the stratification that we have just constructed for $\mathcal{F}(4)$. Recall that each stratum is an orbit of the action of the subgroup of the general linear group that fixes the point $v$, the line $l$, and hence, the plane $p$ (see notation in Section III): $G=\left\{\mathbf{g} \in G L_{4}(\mathbb{R}) \mid \mathbf{g}(v)=v, \mathbf{g}(l)=l\right\}$. Hence, we will construct a representative element in each orbit $\mathcal{O}_{(\omega, \Delta)}$, which will be called standard flag and will be denoted by $C_{\bullet}^{(\omega, \Delta)}$, and transform it to all the other flags of the same orbit by the action of the linear subgroup $G$.

Once a basis $\left\{\mathbf{e}_{1}, \mathbf{e}_{2}, \mathbf{e}_{3}, \mathbf{e}_{4}\right\}$ satisfying $l=\left\langle\mathbf{e}_{1}, \mathbf{e}_{2}\right\rangle$ and $v=$ $\left\langle\mathbf{e}_{3}\right\rangle$ is fixed, the matrix representation of any element $g$ of $G$ is

$$
\mathbf{g}=\left(\begin{array}{cccc}
g_{11} & g_{12} & 0 & g_{14} \\
g_{21} & g_{22} & 0 & g_{24} \\
0 & 0 & g_{33} & g_{34} \\
0 & 0 & 0 & g_{44}
\end{array}\right)
$$

where the $i$ th column of the matrix is the image by $\mathbf{g}$ of $\mathbf{e}_{i}$. Since $\mathrm{g}$ is invertible, the determinant of the matrix is nonzero

$$
\operatorname{det}(\mathbf{g})=\operatorname{det}\left(\begin{array}{ll}
g_{11} & g_{12} \\
g_{21} & g_{22}
\end{array}\right) g_{33} g_{44} \neq 0
$$

i.e.,

$$
g_{33} \neq 0 \quad g_{44} \neq 0 \quad \text { and } \quad \operatorname{det}\left(\begin{array}{ll}
g_{11} & g_{12} \\
g_{21} & g_{22}
\end{array}\right) \neq 0 .
$$

We adapt from Magyar [32] a method to construct a triplet $\left(Z, Y_{\bullet}, C_{\bullet}\right)$ in each orbit of $\mathbb{P}^{3} \times \mathcal{F}(\mathbf{b}) \times \mathcal{F}(4)$ from a suitable basis $V$. We will choose a basis $V$ for each decorated permutation (satisfying the hypothesis of Theorem 1) in order that the obtained triplet is contained in $j(\mathcal{F}(4))$, namely in each constructed triplet $\left(Z, Y_{\bullet}, C_{\bullet}\right)$, we will recover the fixed pair $Z=v$ and $Y_{\bullet}=(0 \subset l \subset p \subset E)$. Given a decorated permutation $(\omega, \Delta)$ that represents a stratum $\mathcal{O}_{(\omega, \Delta)}$ in $\mathcal{F}(4)$, we define a vector $\mathbf{u}$ depending on the type of decoration

$$
\mathbf{u}= \begin{cases}\mathbf{e}_{3}, & \text { if } 2 \text { is the only decorated value of } \omega \\ & \text { like }(\omega, \Delta)=3 \underline{2} 11 \\ \mathbf{e}_{3}-\mathbf{e}_{1}, & \text { if } 2 \text { and the first } 1 \text { are the only } \\ & \text { decorated values of } \omega, \text { like }(\omega, \Delta)=3 \underline{211} \\ \mathbf{e}_{3}-\mathbf{e}_{2}, & \text { if } 2 \text { and the second } 1 \text { are the only } \\ \text { decorated values of } \omega, \text { like }(\omega, \Delta)=3 \underline{2} 1 \underline{1} .\end{cases}
$$

Since $\left\{\mathbf{e}_{1}, \mathbf{e}_{2}, \mathbf{u}, \mathbf{e}_{4}\right\}$ are linearly independent, we are able, using these vectors, to construct a basis $V=$ $\left(\mathbf{v}_{\omega(1) 1}, \mathbf{v}_{\omega(2) 2}, \mathbf{v}_{\omega(3) 3}, \mathbf{v}_{\omega(4) 4}\right)$, which is indexed according to $(\omega, \Delta)$ as follows: $\mathbf{e}_{1}$ and $\mathbf{e}_{2}$ correspond to the vectors $\mathbf{v}_{\omega(j) j}$ with $\omega(j)=1, \mathbf{u}$ is assigned to the vector $\mathbf{v}_{\omega(j) j}$ with $\omega(j)=2$, and $\mathbf{e}_{4}$ corresponds to the vector $\mathbf{v}_{\omega(j) j}$ with $\omega(j)=3$. In conclusion, sorting $\mathrm{V}$ by its first subindex, we have $\left(\mathbf{v}_{1 a}, \mathbf{v}_{1 b}, \mathbf{v}_{2 c}, \mathbf{v}_{3 d}\right)=\left(\mathbf{e}_{1}, \mathbf{e}_{2}, \mathbf{u}, \mathbf{e}_{4}\right)$.

Using this basis $V$, which is indexed as we have just described, we assign to the decorated permutation $(\omega, \Delta)$ the following triplet $\left(Z, Y_{\bullet}, C_{\bullet}\right)$, where $Y_{\bullet}=\left(0 \subset Y_{1} \subset Y_{2} \subset\right.$ $\left.Y_{3}=E\right), C_{\bullet}=\left(0 \subset C_{1} \subset C_{2} \subset C_{3} \subset C_{4}=E\right)$ with $Y_{i}:=$ $\left\langle\mathbf{v}_{i^{\prime} j} \mid i^{\prime} \leq i\right\rangle, Z:=\left\langle\sum_{j \in \Delta} \mathbf{v}_{\omega(j) j}\right\rangle$ and $C_{j}:=\left\langle\mathbf{v}_{i j^{\prime}} \mid j^{\prime} \leq j\right\rangle$, thus resulting in $Z=\left\langle\mathbf{e}_{3}\right\rangle=v$ and

$$
\begin{aligned}
Y_{\bullet} & =\left(0 \subset\left\langle\mathbf{e}_{1}, \mathbf{e}_{2}\right\rangle=l \subset\left\langle\mathbf{e}_{1}, \mathbf{e}_{2}, \mathbf{u}\right\rangle\right. \\
& \left.=\left\langle\mathbf{e}_{1}, \mathbf{e}_{2}, \mathbf{e}_{3}\right\rangle=p \subset\left\langle\mathbf{e}_{1}, \mathbf{e}_{2}, \mathbf{u}, \mathbf{e}_{4}\right\rangle=E\right)
\end{aligned}
$$

which both remain fixed for any $(\omega, \Delta)$ and comprise the desired features $v, l$, and $p$, as desired, and

$$
\begin{aligned}
C_{\bullet}^{(\omega, \Delta)}: & =C_{\bullet}=\left(0 \subset\left\langle\mathbf{v}_{\omega(1) 1}\right\rangle \subset\left\langle\mathbf{v}_{\omega(1) 1}, \mathbf{v}_{\omega(2) 2}\right\rangle\right. \\
& \left.\subset\left\langle\mathbf{v}_{\omega(1) 1}, \mathbf{v}_{\omega(2) 2}, \mathbf{v}_{\omega(3) 3}\right\rangle \subset E\right)
\end{aligned}
$$

which is called the standard flag of the orbit $O_{(\omega, \Delta)}$. The whole $G$-orbit corresponding to $(\omega, \Delta)$ consists of all the flags obtained by applying the group $G$ to the standard flag

$$
\mathcal{O}_{(\omega, \Delta)}=\left\{\mathbf{g} C_{\bullet}^{(\omega, \Delta)} \mid \mathbf{g} \in G\right\} \subset \mathcal{F}(4) .
$$

Any (complete) flag $C_{\bullet}=\left(0 \subset C_{1} \subset C_{2} \subset C_{3} \subset C_{4}=E\right)$ can be represented by an $n \times n$ matrix: the first $i$ columns spanning the subspace $C_{i}$, assuming each column as homogeneous coordinates of a point in $\mathbb{P}^{3}=\mathbb{P}(E)$. Any such matrix will be called a matrix representation of the flag $C_{\bullet}$ and will be written between square brackets, in contrast to the matrix representation of a linear application, between parentheses. Note that a matrix representation is not unique, since the matrix obtained by multiplying a column by a nonzero scalar or adding a linear combination of previous columns represents the same flag. 
Hence, a matrix representation of $C_{\bullet}^{(\omega, \Delta)}$ is given by

$$
C_{\bullet}^{(\omega, \Delta)}=\left[\begin{array}{llll}
\mathbf{v}_{\omega(1) 1} & \mathbf{v}_{\omega(2) 2} & \mathbf{v}_{\omega(3) 3} & \mathbf{v}_{\omega(4) 4}
\end{array}\right]
$$

and a matrix representation of the flag $\mathbf{g} C_{\bullet}^{(\omega, \Delta)}$ is obtained by applying $\mathbf{g}$ to the vectors of $C_{\bullet}^{(\omega, \Delta)}$ or, in matrix formulation, by multiplying the matrix of $\mathbf{g}$ by the matrix representation of $C_{\bullet}^{(\omega, \Delta)}$. A characterization of the stratum $\mathcal{O}_{(\omega, \Delta)}$ is a finite collection of matrix representations (with variable entries), which together represent all the flags in this stratum. A characterization of $\mathcal{O}_{(\omega, \Delta)}$ can be given by a unique matrix representation, as for instance, the product of the matrix (with variable entries) of $\mathbf{g}$ as in (1) and a matrix representation of the standard flag. Sometimes, however, especially when making explicit the number of DOFs of the stratum, some simplifications will be performed, and several cases shall be distinguished, and then, more than one matrix representation will be needed to cover all the flags in the stratum, as the following examples show.

1) Example: Here, we will find all the configurations of the stratum $\mathcal{O}_{(\omega, \Delta)}$ with $(\omega, \Delta)=13 \underline{21}$. Since the second 1 is decorated $\mathbf{u}=\mathbf{e}_{3}-\mathbf{e}_{2}$ and the vectors of the basis $V$ are $\left(\mathbf{v}_{11}, \mathbf{v}_{32}, \mathbf{v}_{23}, \mathbf{v}_{14}\right)=\left(\mathbf{e}_{1}, \mathbf{e}_{4}, \mathbf{e}_{3}-\mathbf{e}_{2}, \mathbf{e}_{2}\right)$. The matrix representation of the standard flag $C_{\bullet}^{1321}$ is

$$
C_{\bullet}^{13 \underline{21}}=\left(\begin{array}{cccc}
1 & 0 & 0 & 0 \\
0 & 0 & -1 & 1 \\
0 & 0 & 1 & 0 \\
0 & 1 & 0 & 0
\end{array}\right)
$$

Any flag $C$. generated by the action of $G$ is of the form

$$
\begin{aligned}
C_{\bullet}= & \mathbf{g}\left(C_{\bullet}^{13 \underline{21}}\right) \\
= & \left(\begin{array}{cccc}
g_{11} & g_{12} & 0 & g_{14} \\
g_{21} & g_{22} & 0 & g_{24} \\
0 & 0 & g_{33} & g_{34} \\
0 & 0 & 0 & g_{44}
\end{array}\right)\left[\begin{array}{cccc}
1 & 0 & 0 & 0 \\
0 & 0 & -1 & 1 \\
0 & 0 & 1 & 0 \\
0 & 1 & 0 & 0
\end{array}\right] \\
= & {\left[\begin{array}{cccc}
g_{11} & g_{14} & -g_{12} & g_{12} \\
g_{21} & g_{24} & -g_{22} & g_{22} \\
0 & g_{34} & g_{33} & 0 \\
0 & g_{44} & 0 & 0
\end{array}\right] }
\end{aligned}
$$

with

$$
g_{33} \neq 0, \quad g_{44} \neq 0, \quad \operatorname{det}\left(\begin{array}{ll}
g_{11} & g_{12} \\
g_{21} & g_{22}
\end{array}\right) \neq 0 .
$$

Performing some simplifications (adding the third column to the fourth, and dividing the second, third, and fourth columns by $g_{44}, g_{33}$, and $g_{33}$, respectively) and using $*$ to mean any real number and $\sharp$ any nonzero real number

$$
C_{\bullet}=\left[\begin{array}{llll}
a & * & c & 0 \\
b & * & d & 0 \\
0 & * & 1 & 1 \\
0 & 1 & 0 & 0
\end{array}\right], \quad \text { with } \operatorname{det}\left(\begin{array}{ll}
a & c \\
b & d
\end{array}\right) \neq 0
$$

We can subtract $r$ times the first column from the third column. Taking $r=(a c+b d) /\left(a^{2}+b^{2}\right)$, the scalar product of $(a, b)$ and $(c, d)$, the resulting vector can be expressed

$$
\begin{aligned}
& \text { as }-b t \mathbf{e}_{1}+a t \mathbf{e}_{2}+\mathbf{e}_{3} \text {, and } \\
& C \bullet \cdot\left[\begin{array}{cccc}
a & * & -t b & 0 \\
b & * & t a & 0 \\
0 & * & 1 & 1 \\
0 & 1 & 0 & 0
\end{array}\right] \text {, with } t \neq 0 \text {, and }(a, b) \neq(0,0) .
\end{aligned}
$$

Considering separately the cases $a \neq 0$ and $b \neq 0$, we have a characterization of the stratum covered by the two matrix representations (depending each one on four parameters)

$$
C \bullet ⿻ \begin{cases}{\left[\begin{array}{llll}
1 & 0 & 0 & 0 \\
* & * & \sharp & 0 \\
0 & * & 1 & 1 \\
0 & 1 & 0 & 0
\end{array}\right],} & \text { if } a \neq 0 \\
{\left[\begin{array}{llll}
* & * & \sharp & 0 \\
1 & 0 & 0 & 0 \\
0 & * & 1 & 1 \\
0 & 1 & 0 & 0
\end{array}\right],} & \text { if } b \neq 0 .\end{cases}
$$

Hence, $\mathcal{O}_{1321}$ has dimension 4 (cf., Fig. 3).

2) Example: If $(\omega, \Delta)=3 \underline{21} 1$, then the standard flag and characterization of this stratum are, respectively

$$
\begin{gathered}
C_{\bullet}^{3 \underline{21} 1}=\left[\begin{array}{cccc}
0 & -1 & 1 & 0 \\
0 & 0 & 0 & 1 \\
0 & 1 & 0 & 0 \\
1 & 0 & 0 & 0
\end{array}\right] \\
C_{\bullet}=\mathbf{g}\left(C_{\bullet}^{3 \underline{211}}\right)=\left[\begin{array}{cccc}
g_{14} & -g_{11} & g_{11} & g_{12} \\
g_{24} & -g_{21} & g_{21} & g_{22} \\
g_{34} & g_{33} & 0 & 0 \\
g_{44} & 0 & 0 & 0
\end{array}\right]
\end{gathered}
$$

i.e.,

$$
C .=\left[\begin{array}{cccc}
* & a & 0 & -b \\
* & b & 0 & a \\
* & 1 & 1 & 0 \\
1 & 0 & 0 & 0
\end{array}\right], \quad \text { with }(a, b) \neq(0,0) .
$$

The following equivalent characterization proves that $\mathcal{O}_{3211}$ is a submanifold of dimension 5 [cf., Fig. 3(b)]:

$$
C .=\left\{\begin{array}{lll}
{\left[\begin{array}{llll}
* & 1 & 0 & 0 \\
* & * & 0 & 1 \\
* & \sharp & 1 & 0 \\
1 & 0 & 0 & 0
\end{array}\right],} & \text { if } a \neq 0 \\
{\left[\begin{array}{cccc}
* & * & 0 & 1 \\
* & \sharp & 0 & 0 \\
* & 1 & 1 & 0 \\
1 & 0 & 0 & 0
\end{array}\right],} & \text { if } b \neq 0 .
\end{array}\right.
$$

\section{APPENDIX C}

\section{Stratification OF THE SET OF AfFine Flags $\mathcal{F}_{\mathcal{A}}(4)$}

In this part of the Appendix, we will show in detail the subdivision and the adjacency relations between the principal strata when they are restricted to $\mathcal{F}_{\mathcal{A}}(4)$, namely the strata of dimensions 6 and 5, since they represent the nonsingular configurations and the most general singular configurations, respectively.

In our application, the fixed pair $\left(Z, Y_{\bullet}\right)$ is attached to the base of the robot; therefore, when choosing an affine coordinate system (which is equivalent to choosing the plane at infinity 
$\Pi_{\infty}$ ), the subspaces composing $Z$ and $Y_{\bullet}$ must be affine. Hence, we have chosen $Z=\left\langle\mathbf{e}_{3}\right\rangle$ and $Y_{\bullet}=\left(\{0\} \subset\left\langle\mathbf{e}_{1}, \mathbf{e}_{2}\right\rangle \subset\right.$ $\left.\left\langle\mathbf{e}_{1}, \mathbf{e}_{2}, \mathbf{e}_{3}\right\rangle \subset E\right), \Pi_{\infty}=\left\{\left[x_{1}, x_{2}, x_{3}, x_{4}\right] \in \mathbb{P}^{3} \mid x_{1}-x_{3}=\right.$ $0\}$, which satisfy these restrictions.

In $\mathcal{F}(4)$, the only stratum of dimension 6 is $\mathcal{O}_{32} 1 \underline{1}$, and its standard flag and characterization are, respectively

$$
\begin{array}{r}
{\left[\begin{array}{cccc}
0 & 0 & 1 & 0 \\
0 & -1 & 0 & 1 \\
0 & 1 & 0 & 0 \\
1 & 0 & 0 & 0
\end{array}\right]} \\
\text { with } \operatorname{det}\left(\begin{array}{cc}
a & b \\
c & d
\end{array}\right) \neq 0 .
\end{array}
$$

$\mathcal{O}_{3211}$ is connected and, despite having seven parameters, if the third column is multiplied by a nonzero scalar, the same flag is represented, and therefore, it has dimension 6 . In $\mathcal{F}_{\mathcal{A}}(4)$, with the restriction $x_{1} \neq x_{3}$, it splits into the following two connected components:

$\mathcal{O}^{+}=\left\{\right.$flags with $\left.x_{1}<x_{3}\right\}, \quad \mathcal{O}^{-}=\left\{\right.$flags with $\left.x_{1}>x_{3}\right\}$.

In $\mathcal{F}(4)$, this 6-D stratum is adjacent to three strata of dimension 5, namely $\mathcal{O}_{2311}, \mathcal{O}_{3121}$, and $\mathcal{O}_{3211}$.

$\mathcal{O}_{2311}$ represents the singularities caused by a null volume of the first tetrahedron; the point of the platform touches the plane of the base $\left(v^{*}-p\right)$. Its standard flag and characterization are, respectively

$$
\begin{array}{rr}
{\left[\begin{array}{cccc}
0 & 0 & 1 & 0 \\
-1 & 0 & 0 & 1 \\
1 & 0 & 0 & 0 \\
0 & 1 & 0 & 0
\end{array}\right]} & \text { and }\left[\begin{array}{cccc}
c & x_{1} & a & 0 \\
d & x_{2} & b & 0 \\
1 & x_{2} & 0 & 1 \\
0 & 1 & 0 & 0
\end{array}\right] \\
& \text { with } \operatorname{det}\left(\begin{array}{ll}
a & b \\
c & d
\end{array}\right) \neq 0 .
\end{array}
$$

It is connected in $\mathcal{F}(4)$, and in $\mathcal{F}_{\mathcal{A}}(4)$, it splits into $\mathcal{O}_{v^{*}-p}^{+}$ and $\mathcal{O}_{v^{*}-p}^{-}$, which comprise the flags with $c<1$ and $c>1$, respectively.

$\mathcal{O}_{3121}$ represents the singularities corresponding to a zero determinant of the second tetrahedron: the lines of the base and platform intersect $\left(l^{*} \cdot l\right)$. Its standard flag and characterization are, respectively

$$
\begin{aligned}
& {\left[\begin{array}{cccc}
0 & 1 & 0 & 0 \\
0 & 0 & -1 & 1 \\
0 & 0 & 1 & 0 \\
1 & 0 & 0 & 0
\end{array}\right] \quad \text { and } \quad\left[\begin{array}{cccc}
x_{1} & a & c & 0 \\
x_{2} & b & d & 0 \\
x_{3} & 0 & 1 & 1 \\
1 & 0 & 0 & 0
\end{array}\right]} \\
& \text { with } \operatorname{det}\left(\begin{array}{ll}
a & b \\
c & d
\end{array}\right) \neq 0 \text {. }
\end{aligned}
$$

Despite having seven parameters, the two transformations $(a, b) \mapsto(\lambda a, \lambda b)$, for any nonzero $\lambda$, and $(c, d) \mapsto(c+\mu a, d+$ $\mu b)$, for any $\mu$, do not change the represented flag and the resulting dimension is 5 . In the affine flag manifold, this stratum splits into $\mathcal{O}_{l^{*} l}^{+}$and $\mathcal{O}_{l^{*} \cdot l}^{-}$, which comprise the flags with $x_{1}<x_{3}$ and $x_{1}>x_{3}$, respectively.

The last 5-D strata of $\mathcal{F}(4), \mathcal{O}_{3211}$, corresponds to the singularities of the kind $\left(v-p^{*}\right)$, the vertex of the basis is contained in the plane of the platform, and the volume of the third tetrahedron vanishes. The standard flag and the characterization of this stratum are, respectively

$$
\begin{aligned}
& {\left[\begin{array}{cccc}
0 & -1 & 1 & 0 \\
0 & 0 & 0 & 1 \\
0 & 1 & 0 & 0 \\
1 & 0 & 0 & 0
\end{array}\right] \quad \text { and } \quad\left[\begin{array}{cccc}
x_{1} & a & 0 & -b \\
x_{2} & b & 0 & a \\
x_{3} & 1 & 1 & 0 \\
1 & 0 & 0 & 0
\end{array}\right]} \\
& \text { with }(a, b) \neq(0,0) \text {. }
\end{aligned}
$$

Restricted to $\mathcal{F}_{\mathcal{A}}(4)$, it also splits into two strata $\mathcal{O}_{v-p^{*}}^{+}$and $\mathcal{O}_{v-p^{*}}^{-}$, with the same restrictions as the previous one: $x_{1}<x_{3}$ and $x_{1}>x_{3}$, respectively.

Next, we will show how the Bruhat order introduced in Section III-A for $\mathcal{F}(4)$ needs to be refined in order to determine the adjacency relations between the strata of $\mathcal{F}_{\mathcal{A}}(4)$ (see Fig. 6).

In $\mathcal{F}_{\mathcal{A}}(4)$ not all the boundary conditions [that related two strata of $\mathcal{F}(4)$ ] are preserved. Consider a family $C_{\bullet}(\tau)$ of complete flags lying on $\mathcal{O}^{+}$for $\tau \neq 0$. Since the first subspace can be expressed as

$$
C_{1}(\tau)=\left\langle x_{1}(\tau) \mathbf{e}_{1}+x_{2}(\tau) \mathbf{e}_{2}+x_{3}(\tau) \mathbf{e}_{3}+\mathbf{e}_{4}\right\rangle
$$

with $x_{1}(\tau)<x_{3}(\tau)$, the $C \bullet(\tau)$ cannot converge, for $\tau=0$ to a flag belonging to $\mathcal{O}_{l^{*}, l}^{-}$or to $\mathcal{O}_{v-p^{*}}^{-}$, since these strata have the restriction $x_{1}>x_{3}$. The same argument applies to show that $\mathcal{O}_{l^{*} \cdot l}^{+}$and $\mathcal{O}_{v-p^{*}}^{+}$are not in the adherence of $\mathcal{O}^{-}$. Since $\mathcal{O}_{3121} \subset \overline{\mathcal{O}}_{3211}$, we know that $\mathcal{O}_{l^{*}, l}^{+} \subset \overline{\mathcal{O}}^{+} \cup \overline{\mathcal{O}}^{-}$, and we can conclude that $\mathcal{O}_{l^{*} l}^{+} \subset \overline{\mathcal{O}}^{+}$. Analogous arguments apply for $\mathcal{O}_{l^{*} \cdot l}^{-}$, $\mathcal{O}_{v-p^{*}}^{+}$, and $\mathcal{O}_{v-p^{*}}^{-}$. On the other hand, by using suitable families of flags, it has been proved in [24] that $\mathcal{O}_{v^{*}-p}^{+} \subset \overline{\mathcal{O}}^{+}$, $\mathcal{O}_{v^{*}-p}^{+} \subset \overline{\mathcal{O}}^{-}, \mathcal{O}_{v^{*}-p}^{-} \subset \overline{\mathcal{O}}^{+}$, and $\mathcal{O}_{v^{*}-p}^{-} \subset \overline{\mathcal{O}}^{-}$. This gives all the adjacency relations between the strata of dimensions 6 and 5 of the stratification of $\mathcal{F}_{\mathcal{A}}(4)$ that we have constructed, which are summarized in Fig. 6.

\section{REFERENCES}

[1] H. Li, C. M. Gosselin, M. J. Richard, and B. M. St-Onge, "Analytic form of the six-dimensional singularity locus of the general Gough-Stewart platform," J. Mech. Design, vol. 128, no. 1, pp. 279-287, 2006.

[2] J.-P. Merlet, Parallel Robots. New York: Springer-Verlag, 2000.

[3] A. Wolf and M. Shoham, "Investigation of parallel manipulators using linear complex approximation," Trans. ASME, J. Mech. Design, vol. 125, pp. 564-572, 2003.

[4] B. Dasgupta and T. S. Mruthyunjaya, "The Stewart platform manipulator A review," Mech. Mach. Theory, vol. 35, no. 1, pp. 15-40, 2000.

[5] G. Liu, Y. Lou, and Z. Li, "Singularities of parallel manipulators: A geometric treatment," IEEE Trans. Robot. Autom., vol. 19, no. 4, pp. 579594, Aug. 2003.

[6] I.-M. Chen, J. Angeles, Theingi, and C. Li, "Shaping singularity loci of parallel manipulators using joint-coupling," in Proc. 7th Int. Conf. Autom. Technol., Chiayi, Taiwan., 2003, pp. 515-520.

[7] C. L. Collins and J. M. McCarthy, "The singularity loci of two triangular parallel manipulators," in Proc. IEEE Int. Conf. Adv. Robot., 1997 pp. 473-479.

[8] D. M. Downing, A. E. Samuel, and K. H. Hunt, "Identification of the special configurations of the octahedral manipulator using the pure condition," Int. J. Robot. Res., vol. 21, no. 2, pp. 147-160, 2002.

[9] Ch. Zhang and S.-M. Song, "Forward kinematics of a class of parallel (Stewart) platforms with closed-form solutions," in Proc. Int. Conf. Robot. Autom., 1991, pp. 2676-2681. 
[10] K. H. Hunt and E. J. F. Primrose, "Assembly configurations of some inparallel-actuated manipulators," Mech. Mach. Theory, vol. 28, no. 1, pp. 31-42, 1993.

[11] H. Bruyninckx, "Forward kinematics for Hunt-Primrose parallel manipulators," Mech. Mach. Theory, vol. 34, pp. 657-664, 1999.

[12] S.-K. Song and D.-S. Kwon, "A tetrahedron approach for a unique closedform solution of the forward kinematics of six-dof parallel mechanisms with multiconnected joints," J. Robot. Syst., vol. 19, no. 6, pp. 269-281, 2002.

[13] M. Alberich-Carramiñana, F. Thomas, and C. Torras, "Flagged parallel manipulators," IEEE Trans. Robot., vol. 23, no. 5, pp. 1013-1023, Oct. 2007.

[14] J.-P. Merlet, "Redundant parallel manipulators," Lab. Robot. Autom., vol. 8, no. 1, pp. 17-24, 1996.

[15] B. Dasgupta and T. S. Mruthyunjaya, "Force redundancy in parallel manipulators: Theory and practical issues," Mech. Mach. Theory, vol. 33, no. 6, pp. 727-742, 1998.

[16] L. Notash and R. Podhorodeski, "Forward displacements analysis and uncertainty configurations of parallel manipulators with a redundant branch," J. Robot. Syst., vol. 13, no. 9, pp. 587-601, 1996.

[17] S. Kock and W. Schumacher, "A parallel $x-y$ manipulator with actuation redundancy for high-speed and active-stiffness applications," in Proc. Int. Conf. Robot. Autom., 1998, pp. 2295-2300.

[18] P. A. Voglewede, "Measuring closeness to singularities of parallel manipulators with application to the design of redundant actuation," Ph.D. dissertation, Georgia Inst. Technol., Atlanta, GA, 2004.

[19] I. Bonev, J. Ryu, S.-G. Kim, and S.-K. Lee, "A closed-form solution to the direct kinematics of nearly general parallel manipulators with optimally located three linear extra sensors," IEEE Trans. Robot., vol. 17, no. 2, pp. 148-156, Apr. 2001.

[20] M. Griffis and J. Duffy, "A forward displacement analysis of a class of Stewart platforms," J. Robot. Syst., vol. 6, no. 6, pp. 703-720, 1989.

[21] I. D. Akçali and H. Mutlu, "A novel approach in the direct kinematics of Stewart platform mechanisms with planar platforms," J. Mech. Design, vol. 128, no. 1, pp. 252-263, 2006.

[22] C. Gosselin and J. Angeles, "Singularity analysis of closed-loop kinematic chains," IEEE Trans. Robot., vol. 6, no. 3, pp. 281-290, 1990.

[23] C. Torras, F. Thomas, and M. Alberich-Carramiñana, "Stratifying the singularity loci of a class of parallel manipulators," IEEE Trans. Robot., vol. 22, no. 1, pp. 23-32, Feb. 2006.

[24] M. Garolera, "Study of the singularities of a family of parallel manipulators," Master's thesis, Jun. 2007 [Online]. Available: http://wwwiri.upc.es/people/torras/articles/Garolera07/ (text and videos).

[25] W. Fulton, "Young tableaux," in London Mathematical Society Student Texts, vol. 35, Cambridge, U.K.: Cambridge Univ. Press, 1997.

[26] H. Hiller, "Geometry of Coxeter groups," in Research Notes in Mathematics. London, U.K.: Pitman, 1982.

[27] D. Monk, "The geometry of flag manifolds," in Proc. London Math. Soc., vol. 9, no. 9, pp. 253-286, 1959.

[28] C. Kosniowski, A First Course in Algebraic Topology. Cambridge, U.K.: Cambridge Univ. Press, 1980.

[29] J. Borràs, F. Thomas, and C. Torras, "Analysing the singularities of 6-SPS parallel robots using virtual legs," presented at the 2nd Int. Workshop Fundam. Issues Future Res. Directions Parallel Mechanisms Manipulators, Montpellier, France, Sep. 2008.

[30] P. Ben-Horin and M. Shoham, "Singularity condition of six-degree-offreedom three-legged parallel robots based on Grassmann-Cayley algebra," IEEE Trans. Robot., vol. 22, no. 4, pp. 577-590, Aug. 2006.

[31] E. Smirnov, "Bruhat order for two subspaces and a flag," arXiv:0704.3061, 2007 (Arxiv preprint).

[32] P. Magyar, "Bruhat order of two flags and a line," J. Algebraic Combinatorics, vol. 21, pp. 71-101, 2005.

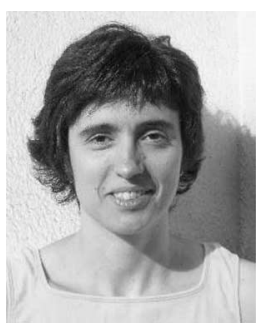

Maria Alberich-Carramiñana received the M.Sc. and the Ph.D. degrees in mathematics, both from the Universitat de Barcelona, Barcelona, Spain, in 1994 and 2000, respectively.

In 2001, she joined the Universitat Politècnica de Catalunya (UPC), Barcelona, as a "Ramón y Cajal" Researcher, where she is currently a Professora Agregada. She is the author of the book Geometry of the Plane Cremona Maps (Springer) in the Lecture Notes in Mathematics series. Her current research interests include singularity theory and algebraic geometry and their applications to robotics and computer vision.

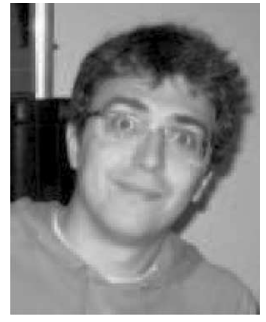

Marçal Garolera received the M.Sc. degree in mathematics and the Engineering degree in telecommunications from the Universitat Politècnica de Catalunya, Barcelona, Spain, in 2006 and 2007, respectively.

In 2006, he was an Intern with the Institut de Robòtica i Informàtica Industrial. Since 2007, he has been with Google, Inc., Dublin, Ireland.

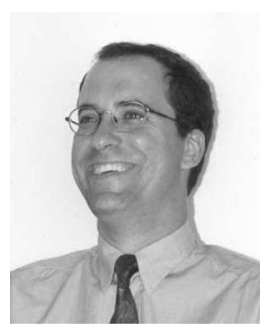

Federico Thomas (M'06) received the Engineering degree in telecommunications in 1984 and the Ph.D. degree (with honors) in computer science in 1988, both from the Universitat Politècnica de Catalunya (UPC), Barcelona, Spain.

During 1999, he was a Visiting Professor, sponsored by the Autonomous Government of Catalonia, with the Oxford University Computing Laboratory, Oxford, U.K. He has been a project leader of several national projects financed by the Spanish Committee for Science and Technology, and by local companies such as ENHER, which is a power-generation company that is now part of ENDESA. He is currently a Research Professor with the Spanish Scientific Research Council, Barcelona. His current research interests include geometry and kinematics with applications to robotics, computer graphics, and computer vision.

Prof. Thomas is an Associate Editor of the IEEE TRANSACTIONS ON ROBOTICS.

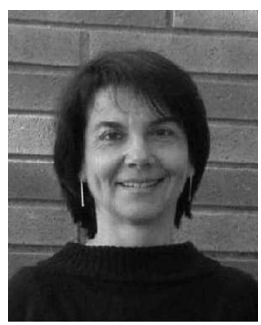

Carme Torras (M'07) received the M.Sc. degree in mathematics from the Universitat de Barcelona, Barcelona, Spain, the M.Sc. degree in computer science from the University of Massachusetts, Amherst, and the Ph.D. degree in computer science from the Universitat Politècnica de Catalunya (UPC), Barcelona.

She has been a local project leader of several European projects, such as Planning RObot Motion, (PROMotion), Robot Control based on Neural Network Systems (CONNY), Self-organization and Analogical Modeling using Subsymbolic Computing, (SUBSYM), Behavioral Learning: Sensing and Acting, (B-LEARN), and the ongoing sixth framework IP project "Perception, Action and COgnition through Learning of Object-Action Complexes" (PACO-PLUS). She is currently a Research Professor with the Spanish Scientific Research Council, Barcelona. She has authored or coauthored five books and about 200 papers in the areas of robot kinematics, geometric reasoning, computer vision, and neurocomputing. 